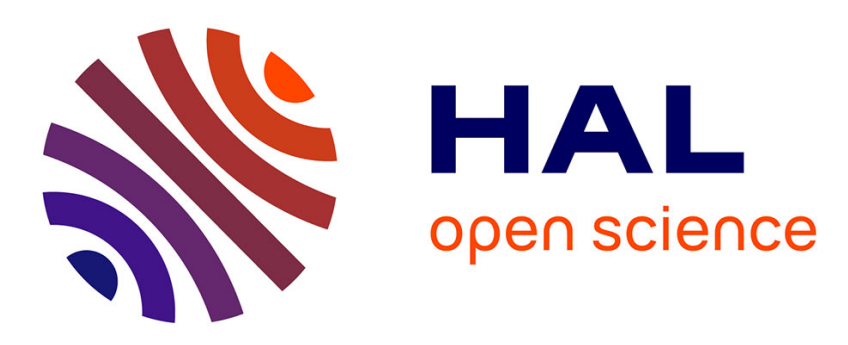

\title{
Structure of aqueous colloidal formulations used in coating and agglomeration processes: Mesoscale model and experiments
}

\author{
Ahmed Jarray, Vincent Gerbaud, Mehrdji Hemati
}

\section{- To cite this version:}

Ahmed Jarray, Vincent Gerbaud, Mehrdji Hemati. Structure of aqueous colloidal formulations used in coating and agglomeration processes: Mesoscale model and experiments. Powder Technology, 2016, 291, pp.244-261. 10.1016/j.powtec.2015.12.033 . hal-01338896

\section{HAL Id: hal-01338896 https://hal.science/hal-01338896}

Submitted on 12 Jul 2016

HAL is a multi-disciplinary open access archive for the deposit and dissemination of scientific research documents, whether they are published or not. The documents may come from teaching and research institutions in France or abroad, or from public or private research centers.
L'archive ouverte pluridisciplinaire HAL, est destinée au dépôt et à la diffusion de documents scientifiques de niveau recherche, publiés ou non, émanant des établissements d'enseignement et de recherche français ou étrangers, des laboratoires publics ou privés. 


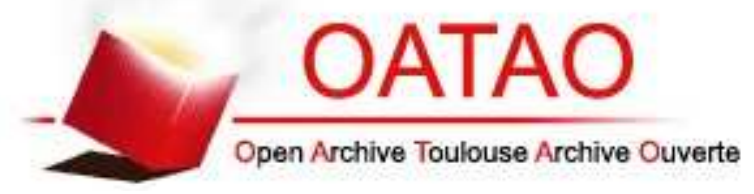

\section{Open Archive TOULOUSE Archive Ouverte (OATAO)}

OATAO is an open access repository that collects the work of Toulouse researchers and makes it freely available over the web where possible.

This is an author-deposited version published in : http://oatao.univ-toulouse.fr/ Eprints ID : 15862

To link to this article : DOI : 10.1016/j.powtec.2015.12.033

URL : http://dx.doi.org/10.1016/j.powtec.2015.12.033

To cite this version : Jarray, Ahmed and Gerbaud, Vincent and

Hemati, Mehrdji Structure of aqueous colloidal formulations used in coating and agglomeration processes: Mesoscale model and

experiments. (2016) Powder Technology, vol. 291. pp. 244-261.

ISSN 0032-5910

Any correspondence concerning this service should be sent to the repository administrator: staff-oatao@ listes-diff.inp-toulouse.fr 


\title{
Structure of aqueous colloidal formulations used in coating and agglomeration processes: Mesoscale model and experiments
}

\author{
A. Jarray *, V. Gerbaud, M. Hemati \\ a Université de Toulouse, INP, UPS, LGC (Laboratoire de Génie Chimique), 4 allée Emile Monso, F-31432 Toulouse Cedex 04, France \\ ${ }^{\mathrm{b}}$ LGC, INP, ENSIACET, 4 Allée Emile Monso, 31432 Toulouse, France
}

Keywords:

Agglomeration

Pharmaceutical products

DPD

Mesoscale simulation

Colloids

Coating

\begin{abstract}
A B S T R A C T
In coating and agglomeration processes, the properties of the final product, such as solubility, size distribution, permeability and mechanical resistance, depend on the process parameters and the binder (or coating) solution properties. These properties include the type of solvent used, the binder composition and the affinity between its constituents.

In this study, we used mesoscale simulations to investigate the structure of agglomerates formed in aqueous colloidal formulations used in coating and granulation processes. The formulations include water, a film forming polymer (Hydroxypropyl-methylcellulose, HPMC), a hydrophobic filler (Stearic acid, SA) and a plasticizer (Polyethylene glycol, PEG). For the simulations, dissipative particle dynamics (DPD) and a coarse-grained approach were used. In the DPD method, the materials are described as a set of soft beads interacting according to the Flory-Huggins model. The repulsive interactions between the beads were evaluated using the solubility parameter $(\delta)$ as input, where $\delta$ was calculated by all-atom molecular dynamics. The DPD simulation results were compared to experimental results obtained by cryogenic-SEM and particle size distribution analysis.

DPD simulation results showed that the HPMC polymer is able to adsorb in depth into the inner core of SA particle and covers it with a thick layer. We also observed that the structure of HPMC-SA mixture varies under different amounts of SA. For high amounts of SA, HPMC is unable to fully stabilize SA. Affinity between the binder materials was deduced from the DPD simulations and compared with Jarray et al. (2014) theoretical affinity model.

Experimental results presented similar trends; particle size distribution analysis showed that for low percentage of SA (below $10 \% \mathrm{w} / \mathrm{w}$ ) and in the presence of HPMC, the majority of SA particles are below $1 \mu \mathrm{m}$ in diameter. Cryogenic-SEM images reveal that SA crystals are covered and surrounded by HPMC polymer. SA crystals remain dispersed and small in size for low percentages of SA.
\end{abstract}

\section{Introduction}

Particle growth process relies on the addition of a solution or suspension which will adhere on particles to produce agglomerate or coated particles. The former is governed by agglomeration mechanisms, where the particles agglomerate by virtue of a binder. The latter is obtained through coating or layering process, where the particles are entirely covered by the coating solution. Whether, it is a coated particle or an agglomerate, the coating solution (or the binder) is usually prepared through aqueous polymer dispersion.

Hydrophilic stabilizing polymers (such as hydroxypropylmethylcellulose, HPMC), plasticizers (such as polyethylene glycol, PEG) or hydrophobic filler (such as stearic acid, SA) are added during the preparation of the polymer dispersion. These additives are present

* Corresponding author at: Université de Toulouse, INP, UPS, LGC (Laboratoire de Génie Chimique), 4 allée Emile Monso, F-31432 Toulouse Cedex 04, France.

E-mail addresses: ahmed.jarray@ensiacet.fr (A. Jarray), vincent.gerbaud@ensiacet.fr (V. Gerbaud), mehrdji.hemati@ensiacet.fr (M. Hemati). in the final binder or coating solution, therefore, they affect various properties of the final product. The film forming dispersions should be physically stable and the hydrophobic particles should be uniformly dispersed in the medium. This can be achieved by formulating the adequate coating or binder solution and by obtaining stable colloids with good affinity and sufficient interactions between its components.

Previous theoretical models to predict binder-substrate and coating components affinity have been published; Rowe [1] and Barra [2] used the solubility parameter to predict the interactions between polymers in binary systems based on a cohesion-adhesion model. Benali [3] adopted the same approach but ran molecular simulations to calculate the solubility parameter. Jarray et al. [4] compared different theoretical predictive approaches in binary systems and generalized the model of cohesion-adhesion for ternary systems, including water.

The above models are effective to predict the components affinity, yet they give limited insights on the stability of the coating solution. Typically, factors which determine the stability of the colloidal particles in the coating solutions are the diffusivity coefficient, the structure factor, adsorption strength and the surface coverage between the 
stabilizing agent and the dispersed particles. Some of this information can be brought by molecular simulations. Considering that the agglomerate materials we are studying have a size between 0.1 and $100 \mu \mathrm{m}$, it is relevant to perform mesoscale simulations, where molecules are represented as polyatomic beads. As the number of degrees of freedom is reduced compared to all-atom simulations, the computational effort is decreased.

In this study, we perform mesoscale simulations using the dissipative particle dynamics (DPD) method to investigate the structure of colloidal polymeric dispersions and the affinity between polymers in aqueous systems. We begin by discussing colloidal stability and we review the principles of the DPD method and the coarse-grain modeling. Then, we build a coarse grain-model and we describe the DPD approach applied to systems made of polymers (HPMC, polyvinylpyrrolidone (PVP) and microcrystalline cellulose (MCC)) in the presence of a plasticizer (PEG) or a hydrophobic filler (SA) in aqueous systems. Finally, we present the results. The effect of percentage of SA on the structure of the HPMC-SA suspension is investigated by DPD. Structure factor that gives insights about the agglomerate structure is analyzed. DPD affinity predictions are compared to those obtained using our former predictive models (tensile approach and work of adhesion approach) [4]. Simulation results are also compared to experimental results obtained by laser diffraction particle size analyzer and by cryogenic-SEM.

\section{Theory and simulation methods}

\subsection{Colloid stability}

Colloidal systems are dispersed phases finely subdivided in a dispersion medium [5]. Particles are said to be colloidal in character if they possess at least one dimension in the size range 1-100 $\mathrm{nm}$. The dispersion of larger particles, whose size is greater than $1 \mu \mathrm{m}$, is usually referred to as a suspension. A colloidal dispersion is said to be stable when the particles remain dispersed over a long time scale (e.g. months or years) [5]. Colloidal particles always undergo Brownian motion and are attracted to each other with long range attractive forces. Consequently, in order to favor colloid stability, it's necessary to create long range repulsion between the colloidal particles. This can be obtained by entrapping colloidal particles with a polymer (steric stabilization). The polymer will generate a layer at the particles surface and prevent their agglomeration (see Fig. 1(b)). An increase in the layer thickness of the polymer around the colloidal particle has been found to improve the colloidal stability [5]. The layer thickness should be at least several nanometers to provide effective stabilization [6]. Koelmans and Overbeek [7] suggested that only if the thickness of the adsorbed layer was comparable in size to the diameter of the dispersed particles could a polymeric steric mechanism provide sufficient protection.

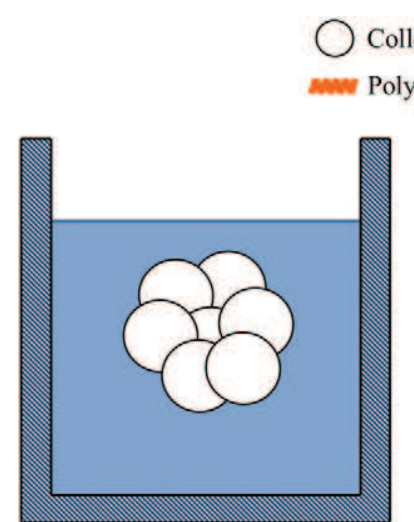

(a)
Colloidal particle Polymer (stabilizing agent)

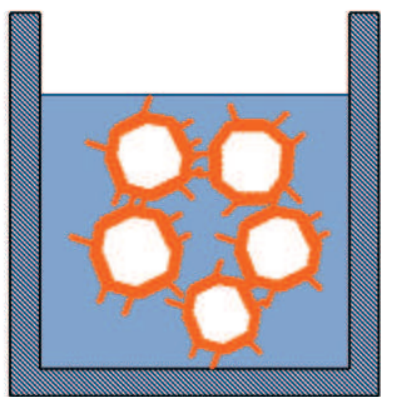

(b)
Fig. 1. Schematic representation of the stabilization of colloidal particles. a) Colloidal particles agglomerate in water, b) Colloidal particles stabilized in water by a polymer.
Walbridge and Waters [8] showed that the minimum steric barrier thickness required for the largest particles was of the order of $5 \mathrm{~nm}$. To attach themselves on the particles, the polymer chains adsorb by affinity on the surface to give full coverage. The attachment between the polymer chains and the colloidal particles should be strong enough to prevent the polymer desorption when the particles undergo Brownian collisions. When the polymer content in the aqueous phase is sufficiently high, the particles may be immobilized in a polymer gel network.

In order to study the colloidal particles stability in aqueous polymeric dispersions, we will use the mesoscale "coarse-grain" approach combined with the dissipative particle dynamics (DPD) simulation method, which is described below.

\subsection{The dissipative particle dynamics (DPD) method: general equations}

The dissipative particle dynamics method (DPD) [9] is a particle mesoscopic simulation method based on the formalism of Langevin with conservation of the momentum [10]. DPD method can be used for the simulation of systems involving colloidal suspensions, emulsions, polymer solutions, Newtonian fluid and polymer melts. In this method, the compounds are composed of molecules described as a set of soft beads that interact dynamically in a continuous space and move along the Newton momentum equation (Eq. (1)). These interactions between the soft beads govern the affinity between the compounds and therefore control the final structure built by the beads in the DPD simulation.

$\frac{d r_{i}}{d t}=v_{i} \quad$ and $\quad \frac{d v_{i}}{d t}=f_{i}$

Where $r_{i}$ and $v_{i}$ are the position and velocity of the bead $i, f_{i}$ represents the sum of the forces acting on the bead:

$f_{i}=\sum_{j \neq i}\left(F_{i j}^{C}+F_{i j}^{D}+F_{i j}^{R}+F_{i j}^{S}\right)$

$F_{i j}$ represent the force exerted by a bead $i$ on the bead $j$. Each bead is subjected to three non-bonded forces; a conservative repulsive force $F^{C}$, that determines the thermodynamic behavior of the system, a dissipative force $F^{D}$, which includes the friction forces, and a random term $F^{R}$, accounting for the omitted degrees of freedom [11], and a bonded force $F^{S}$. The non-bonded forces are given in Eq. (5).

$$
\begin{aligned}
& F_{i j}^{C}=a_{i j} \omega\left(r_{i j}\right) r_{i j} \\
& F_{i j}^{D}=-\partial \omega^{2}\left(r_{i j}\right)\left(r_{i j} \vec{v}_{i j}\right) r_{i j} \\
& F_{i j}^{R}=\sigma(\Delta \mathrm{t})^{-1 / 2} \omega\left(r_{i j}\right) \xi_{i j} r_{i j} \\
& \text { With } \bar{r}_{i j}=r_{i}-r_{j}, r_{i j}=\left|\bar{r}_{i j}\right|, v_{i j}=v_{i}-v_{j}, v_{i j}=v_{i}-v_{j}, r_{i j}=\bar{r}_{i j} / r_{i j} \text { and } a_{i j}=a_{j i} .
\end{aligned}
$$

This last term $a_{i j}$ represents the maximum repulsion between two beads; it encompasses all the physical information of the system. $\partial$ is the parameter of dissipation and $\xi_{i j}$ is a random parameter, which describe the noise with a zero mean and one unit variance. $\omega\left(r_{i j}\right)$ is a weight function which determines the radial dependence of the repulsive force:

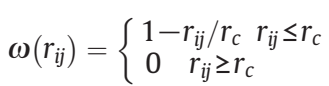

with $r_{c}$ is a cut-off distance. Non-bonded forces act within a sphere of radius $r_{c}$. Outside this sphere, interaction forces are ignored. $\omega\left(r_{i j}\right)$ is qualified as a soft repulsion in opposition to a hard sphere repulsion potential. The soft repulsion fits well the mesoscale nature of the system and allows longer time steps simulations [12]. 
The connected beads are subjected to a bonding spring force:

$F_{i j}^{S}=C_{r} r_{i j}$

with $C_{r}$ as the harmonic spring constant. The behavior of a single polymer chain is deducted by means of interactions generated by its neighbors. DPD method is then applied on soft cluster of molecules called "beads" obtained through "coarse-grain" modeling. The original velocity-Verlet algorithm is used for the integration of all the equations [13].

\subsection{The "coarse-grain" modeling}

In order to reduce the computation cost in molecular simulation for many body systems, we perform simulations at the mesoscopic scale. The molecules or segments of polymer chains are converted into so-called beads through the coarse-grain approach, which consists in aggregating several atoms into a single bead.

Simulations in the DPD system are performed in reduced units. The reduced number density $\bar{\rho}$ in the DPD system is related to the real number density $\rho$ of the compound by the following relationship:

$\bar{\rho}=\rho r_{c}^{3}$

where $\bar{\rho}$ is the number of beads in one cubic simulation cell of volume $r_{c}^{3}$ (see Fig. 2), the cut-off radius $r_{c}$ represents the unit length in the DPD system and also used to establish the reference scale.

Arguments have been raised regarding the difference in the size of beads. The bead volume and mass has no influence on the structure or on the morphology of the simulated system. However, considering that the mass, as well as the volume, is the same for all beads in the DPD system, unit conversion from DPD units to real physical units requires a coarse-grained system with close beads volume and mass [14], especially for cases where the simulation is intended to mimic the real physical quantities and to predict properties such as interfacial energy.

The coarse-graining degree $N_{m}$ represents the number of molecules of water placed in a single bead (see Fig. 2). Grouping several molecules of water in one bead is used to match the volume of the different beads in the DPD simulations. $N_{m}$ can be evaluated using the following formula:

$N_{m}=\frac{\bar{\rho}_{\text {molecule }}}{\bar{\rho}}$

with $\bar{\rho}_{\text {molecule }}$ is the number of molecules in one cubic unit cell of volume $r_{c}^{3}$. The upper script "_" denotes the property (ex. density, surface tension, mass, etc.) in DPD units.
From Eq. (8), the cut-off radius can be obtained by using the following relationship:

$r_{c}=\left(\frac{\bar{\rho}}{\rho}\right)^{1 / 3}=\left(N_{m} V_{\text {molecule }} \bar{\rho}\right)^{1 / 3}$

For a water molecule, we find the same relation proposed by Groot and Rabone [14]:

$r_{c}=3.1072\left(N_{m} \bar{\rho}\right)^{1 / 3}$ in $\AA$

The mass of one bead in the DPD system (i.e. in DPD units) is that of $N_{m}$ water molecules. It can be obtained by the following equation:

$\bar{m}=m_{\text {bead }}=N_{m} m^{m}{ }_{\mathrm{H}_{2} \mathrm{O}}$.

There have been discussions regarding the scalability of the DPD scheme in relation to the upper limit $N_{m}^{\max }$ of the level of coarse-graining. According to Flekkoy et al. [15] and Español et al. [16], grouping many molecules of the same compound into one bead does not change the average kinetic energy of the system. On the other hand, Trovimof [17] stated that the limit of coarsegraining $N_{m}^{\max }$ should not exceed ten molecules of water in a single bead, otherwise, the DPD system will confront the Hansen-Verlet (-Schiff) freezing criterion $[18,19]$. This criterion states that a system congeals when the height of the main peak of the structure factor of the mixture surpasses the quasi-universal value of 2.85 . Such situation must be avoided in DPD simulations. This effect was also observed by Pivkin and Karniadakis [20].

\subsection{DPD parameters calculations}

The forces parameters (in Eqs. (3) and (5)) required to perform the DPD simulations are: the repulsion parameter $a_{i j}$, the parameter of dissipation $\partial$, the random parameter $\xi_{i j}$ and the harmonic spring constant $C_{r}$. There is also the DPD $n_{D P D}$ which represents the number of similar beads by which a polymer chain can be described.

DPD repulsion parameter $a_{i j}$ can be calculated using Hildebrand solubility parameter $\delta$ [21], the number density $\rho$, and the coarse-graining number $N_{m}$. According to Groot and Warren [13], the repulsion parameter $a_{i i}$ that governs the interaction between the beads in the DPD simulation has the following expression:

$a_{i i}=\left(16 N_{m}-1\right) \frac{k_{B} T}{2 \alpha \rho r_{c}^{4}}$
Water bead containing 6 water molecules $\left(N_{m}=6\right)$
Cubic cell containing 3 water beads $(\bar{\rho}=3)$ and 18 water molecules $(\bar{\rho}$
DPD simulation of water $=18)$

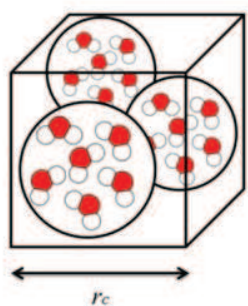

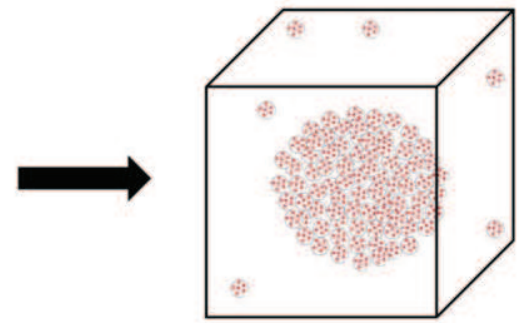

Fig. 2. Schematic representation of the coarse-graining of a water molecule. In this case, $\bar{\rho}=3$ and $N_{m}=6$, the cut-off distance $r_{c}$ is therefore equal to the length of one side of the cubic cell. 


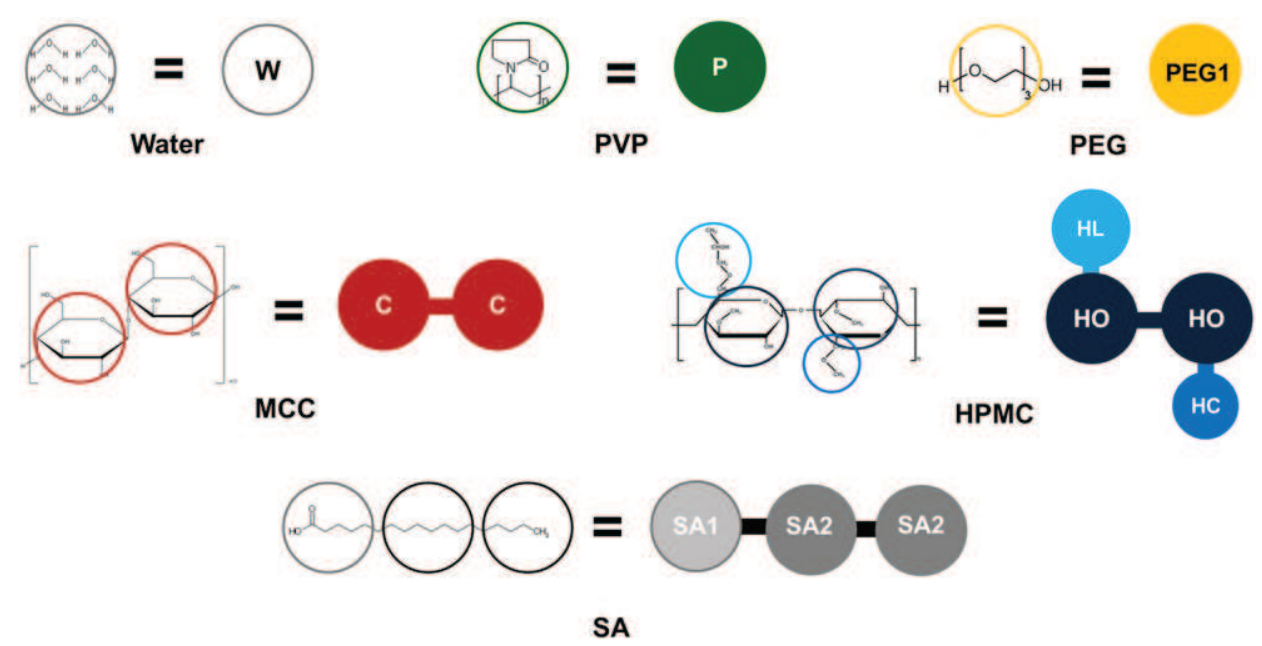

Fig. 3. Coarse-grain method; molecules and monomer conversion into beads for water (W), Polyvinylpyrrolidone (PVP), Polyethylene glycol400 (PEG), Microcrystalline cellulose (MCC), Hydroxypropyl-methylcellulose (HPMC) and Stearic acid (SA).

Were $\alpha$ is an adjustment parameter equal to $0.101( \pm 0.001)$. The detailed demonstration is given in Appendix A. The dimensionless equation takes the following form:

$\bar{a}_{i i}=\frac{\left(16 N_{m}-1\right)}{2 \alpha \bar{\rho}}$

The repulsive parameters for unlike-beads $\bar{a}_{i j}$ can be determined according to a linear relationship with the Flory-Huggins parameter $\chi_{i j}$ $[13,22]$ :

$\bar{a}_{i j}(\bar{\rho}=3)=\bar{a}_{i i}+\frac{\chi_{i j}}{0.286}$

The number density $\bar{\rho}$ is equal to 3 DPD units. This is the value with which the relationship between the repulsion parameter and the FloryHuggins parameter has been established [13].

The Flory-Huggins values can be calculated from the Hildebrand solubility parameter [21] using the formula:

$\chi_{i j}=\frac{\left(\delta_{i}-\delta_{j}\right)^{2}\left(V_{i}+V_{j}\right)}{2 k_{B} T}$

with $V$ the volume of the beads, $\delta_{j}$ and $\delta_{i}$ are the solubility parameters of bead $i$ and $j$ respectively. We notice that the parameter $\bar{a}_{i j}$ is always

Table 1

Solubility parameter and density of repeating units and molecules.

\begin{tabular}{|c|c|c|c|c|c|}
\hline \multicolumn{2}{|c|}{ Compounds } & \multicolumn{2}{|c|}{$\begin{array}{l}\text { Solubility parameter } \delta \\
\text { of the repeating unit } \\
\text { and molecule } \\
\left(\mathrm{J} \cdot \mathrm{cm}^{-3}\right)^{1 / 2}\end{array}$} & \multicolumn{2}{|c|}{$\begin{array}{l}\text { Density } e \text { of the } \\
\text { repeating unit and } \\
\text { molecule } \\
\left(\mathrm{g} \cdot \mathrm{cm}^{-3}\right)\end{array}$} \\
\hline & & COMPASSII & HSPiP & COMPASSII & HSPiP \\
\hline \multicolumn{2}{|l|}{ PVP } & $22.2 \pm 0.3$ & 20.8 & $0.994 \pm 0.02$ & 0.986 \\
\hline \multicolumn{2}{|l|}{ MCC } & $31.5 \pm 0.6$ & 32.0 & $1.347 \pm 0.02$ & 1.434 \\
\hline \multirow[t]{3}{*}{ HPMC } & $\mathrm{HL}$ & $22.1 \pm 0.6$ & 22.4 & $0.893 \pm 0.02$ & 0.918 \\
\hline & $\mathrm{HO}(\times 2)$ & $27.3 \pm 0.3$ & 25.5 & $1.233 \pm 0.01$ & 1.204 \\
\hline & $\mathrm{HC}$ & $18.0 \pm 0.5$ & 17.5 & $0.768 \pm 0.02$ & 0.700 \\
\hline \multirow[t]{2}{*}{ SA } & SA1 & $23.4 \pm 0.3$ & 20.4 & $0.963 \pm 0.01$ & 0.924 \\
\hline & $\mathrm{SA} 2(\times 2)$ & $14.5 \pm 0.2$ & 15.0 & $0.648 \pm 0.01$ & 0.676 \\
\hline PEG1 & PEG1 $(\times 3)$ & $23.7 \pm 0.3$ & 21.4 & $0.992 \pm 0.01$ & 0.993 \\
\hline Water & & $47.5 \pm 0.4$ & $47.8^{\mathrm{a}}$ & $0.962 \pm 0.01$ & $0.997^{\mathrm{a}}$ \\
\hline
\end{tabular}

PVP: Polyvinylpyrrolidone, MCC: Microcrystalline cellulose, HPMC: Hydroxypropyl-methylcellulose, SA: Stearic acid, PEG: Polyethylene glycol.

a HSPiP literature [28]. positive, which means that the conservative force $F^{C}$ is always repulsive.

The previous model of Groot and Warren [13] was built based on the isothermal compressibility of water (See Appendix A) and on the assumption of equal repulsive interactions between similar beads at the interface in binary mixtures $\left(a_{i i}=a_{i j}\right)$. This hypothesis was defended by Groot and Warren [13], and by Maiti and McGrother [23] by saying that all beads have the same cutoff radius and the same volume. In an attempt to eliminate the restriction of having the same repulsive interaction parameters between like beads $\left(a_{i i}=a_{j j}\right)$, Travis et al. [24] recently proposed an alternative relation between conservative interaction parameters $a_{i j}$ and the solubility parameter:

$\left(\delta_{i}-\delta_{j}\right)^{2}=-r_{c}^{4} \alpha\left(\rho_{i}^{2} a_{i i}+\rho_{j}^{2} a_{j j}-2 \rho_{i} \rho_{j} a_{i j}\right)$.

Using the dimensionless parameters: $\quad \bar{a}_{i i}=a_{i i} r_{c} / k_{B} T, \quad \bar{\delta}_{i}=\delta_{i}$ $\left(r_{c}^{3} / k_{B} T\right)^{1 / 2}$ and $\bar{\rho}=\rho r_{c}^{3}$, we obtain the final form of the dimensionless equation:

$\left(\bar{\delta}_{i}-\bar{\delta}_{j}\right)^{2}=-\bar{\rho}^{2} \alpha\left(\bar{a}_{i i}+\bar{a}_{j j}-2 \bar{a}_{i j}\right)$.

Table 2

Conversion of monomer and molecules into beads, and properties of the beads.

\begin{tabular}{|c|c|c|c|c|c|c|}
\hline \multicolumn{2}{|c|}{ Compounds } & \multirow{2}{*}{$\begin{array}{l}M_{w} \text { of the } \\
\text { repeating } \\
\text { unit and } \\
\text { molecule } \\
\left(\mathrm{g} \cdot \mathrm{mol}^{-1}\right)\end{array}$} & \multicolumn{2}{|c|}{ Bead volume $\left(\AA^{3}\right)$} & \multicolumn{2}{|c|}{ Bead radius $(\AA)$} \\
\hline & & & COMPASSII & HSPiP & COMPASSII & HSPiP \\
\hline \multicolumn{2}{|l|}{ PVP } & 111.2 & 185.7 & 187.3 & 3.54 & 3.54 \\
\hline \multicolumn{2}{|l|}{ MCC } & 162.2 & 199.9 & 187.8 & 3.62 & 3.55 \\
\hline \multirow{3}{*}{ HPMC } & $\mathrm{HL}$ & 89.1 & 165.7 & 161.2 & 3.41 & 3.37 \\
\hline & $\mathrm{HO}(\times 2)$ & 144.2 & 194.2 & 198.8 & 3.59 & 3.62 \\
\hline & $\mathrm{HC}$ & 45.1 & 97.5 & 106.9 & 2.58 & 2.95 \\
\hline \multirow[t]{2}{*}{ SA } & SA1 & 115.2 & 198.6 & 207.1 & 3.61 & 3.67 \\
\hline & $\mathrm{SA} 2(\times 2)$ & 85.2 & 218.3 & 209.3 & 3.73 & 3.68 \\
\hline PEG400 & $\begin{array}{l}\text { PEG1 } \\
(\times 3)\end{array}$ & 132.2 & 221.3 & 221.1 & 3.75 & 3.75 \\
\hline Water & & 18.0 & 186.4 & $179.9^{*}$ & 3.54 & 3.50 \\
\hline
\end{tabular}

PVP: Polyvinylpyrrolidone, MCC: Microcrystalline cellulose, HPMC: Hydroxypropyl-methylcellulose, SA: Stearic acid, PEG: Polyethylene glycol.

* HSPiP database [28]. 
Table 3

Number of Beads per chain $n_{\text {DPD }}$ of PVP, MCC and HPMC, calculated using Eq. (20).

\begin{tabular}{llllc}
\hline Composants & $\begin{array}{l}\text { Characteristic } \\
\text { ratio } C_{n}\end{array}$ & $\begin{array}{l}\text { Average molecular } \\
\text { weight } M_{w} \\
\left(\mathrm{~g} \cdot \mathrm{mol}^{-1} \text { ) }\right.\end{array}$ & $\begin{array}{l}\text { Monomer } \\
\text { molecular weight } \\
M_{m} \text { (AMU) }\end{array}$ & $\begin{array}{l}n_{\text {DPD }} \\
\text { (number } \\
\text { of beads) }\end{array}$ \\
\hline PVP & 9.90 & 10000 & 111.2 & 9 \\
MCC & 5.09 & 36000 & 162.2 & 44 \\
HPMC & 4.78 & 20000 & 424.5 & 10 \\
\hline
\end{tabular}

Regarding the conservative interaction parameter between the same beads $a_{i i}$, Travis et al. [24] proposed the following expression:

$a_{i i}=\frac{\delta_{i}^{2}}{\alpha \rho_{i}^{2} r_{c}^{4}}$

In terms of reduced units, Eq. (17) has the following form:

$\bar{a}_{i i}=\frac{\bar{\delta}_{i}^{2}}{\alpha \bar{\rho}_{i}^{2}}$

The fluctuation-dissipation theorem states that the noise parameter $\sigma$ and the dissipation parameter $v$ are connected by the following relation:

$\sigma^{2}=2 \partial k_{B} T$

with $k_{B}$ as the Boltzmann constant. Regarding the parameters of dissipation $\partial$ in Eq. (19), studies have shown that the simulations are not really sensitive to this parameter if it is between 2 and $32 \mathrm{DPD}$ reduced units (i.e. between 0.04019 and $0.64308 \mathrm{~g} \cdot \mathrm{mol}^{-1} \cdot \mathrm{fs}^{-1}$ ) [13]. If this value exceeds 32 DPD units, the force friction between the beads becomes very high and the integration time becomes insufficient to correctly simulate the system. To avoid this problem, an alternative is to decrease the time step.

According to the literature, the harmonic spring constant $C_{r}$ gives good results for values between 2 and 4 DPD units (i.e. between 75 and $150 \mathrm{~J} \cdot \mathrm{mol}^{-1} \cdot \AA^{-2}$ ) [13], which is sufficient to maintain the adjacent beads well-connected in the polymer chain. The spring constant $C_{r}$ is chosen such that the mean distance between connected particles coincides with the peak of the radial distribution function [13].

For polymers, the number of beads $n_{D P D}$ that composes one polymer chain can be estimated with the following equation [25]:

$n_{D P D}=\frac{M_{w}}{M_{m} C_{n}}$

$M_{w}$ is the molecular weight of the polymer, $M_{m}$ the molecular weight of the monomer and $C_{n}$ the characteristic ratio of the polymer.

\section{Experiments and simulation details}

\subsection{Materials}

The compounds chosen in this study are: Polyvinylpyrrolidone (PVP), Microcrystalline cellulose (MCC) (Avicel PH102), Hydroxypropylmethylcellulose (HPMC) (H8384 Sigma), purified Stearic acid (SA), Polyethylene glycol 400 (PEG) and water. All the compounds are purchased from Sigma-Aldrich.

\subsection{Computational simulation details}

\subsubsection{The mesoscale "coarse-grain" model}

As stated earlier, all beads in the DPD simulation should have the same volume; hence, the task of finding the adequate coarse-grained model comprises two concomitant parts; a) estimating the most suited volume common to all beads, and b) avoiding the solidification of the system. In this context, we select the volume of a single bead equal to $180 \AA^{3}$, because, as we will see later, it allows assimilating each molecule or monomer to a bead whose volume is close to that value. Then, a water bead must represent $N_{m}=6$ water molecules (volume of a water molecule $\approx 30 \AA^{3}$ ), which roughly corresponds to a single monomer of PVP and to a single monomer of MCC. SA is thus composed of 3 beads; one bead containing the fragment SA1 and two beads of the fragments SA2 (see Fig. 3). PEG is composed of three similar beads; each one contains the same fragment which we called PEG1. In the same way, HPMC repeating unit is coarse-grained into 4 beads (one HL, two $\mathrm{HO}$ and one $\mathrm{HC}$ ) (see Fig. 3). Irisa and Yokomine [26] and Hongyu Guo et al. [27] also used $N_{m}=6$ water molecules in their DPD simulations.

Since the number density $\bar{\rho}$ is equal to 3 DPD units, a cubic simulation cell with an edge length equal to $r_{c}$ contains three beads with 6 molecules of water each, and corresponds to a volume of $540 \AA^{3}$. Notice that the coarse-graining number $N_{m}=6$ is below the limit specified by Trovimof [17].

\subsubsection{Molecular dynamic simulation and solubility parameter calculation}

Following our previous work [4], the solubility parameters needed to compute the Flory Huggins parameter $\chi$ are calculated using either molecular simulations (in Biovia's Material Studio software product [25]) or Yamamoto's molecular breaking method (HSPiP) [28]. The obtained values are presented in Table 1. All-atom molecular dynamics simulations are performed with an integration step of 1 femtosecond ( $\mathrm{fs}=$ $10^{-15} \mathrm{~s}$ ). The interatomic interactions are described by the COMPASSII (Condensed-phase Optimized Molecular Potentials for Atomistic Simulation Studies) forcefield [29] along with Ewald summation for the long range electrostatics. NPT dynamics is performed first to equilibrate the density of the system for 500 picoseconds ( $\mathrm{ps}=10^{-12} \mathrm{~s}$ ) at room temperature $(T=298 \mathrm{~K})$ and atmospheric pressure $(P=1 \mathrm{~atm})$. Then, another all-atom simulation is launched in the canonical ensemble NVT at a temperature $\mathrm{T}=298 \mathrm{~K}$ for $500 \mathrm{ps}$ in order to track the convergence of the cohesive energy density. The last 50 ps are used for computing the

Table 4

The conservative force parameters $a_{i j}$ and $a_{i i}$ obtained by using Groot and Warren's [13] Eqs. (12) and (13).

\begin{tabular}{|c|c|c|c|c|c|c|c|c|c|c|}
\hline$a_{\mathrm{ij}}$ & & PVP & MCC & HL & $\mathrm{HO}$ & $\mathrm{HC}$ & SA1 & SA2 & PEG1 & Water \\
\hline PVP & & 157.00 & & & & & & & & \\
\hline MCC & & 170.85 & 157.00 & & & & & & & \\
\hline \multirow[t]{3}{*}{ HPMC } & HL & 157.00 & 170.5 & 157.00 & & & & & & \\
\hline & $\mathrm{HO}$ & 161.14 & 159.85 & 161.12 & 157.00 & & & & & \\
\hline & $\mathrm{HC}$ & 159.09 & 179.56 & 158.82 & 167.56 & 157.00 & & & & \\
\hline \multirow[t]{2}{*}{ SA } & SA1 & 157.22 & 167.92 & 157.26 & 159.54 & 160.58 & 157.00 & & & \\
\hline & SA2 & 167.15 & 207.65 & 166.33 & 185.51 & 158.66 & 170.89 & 157.00 & & \\
\hline PEG1 & & 157.38 & 167.64 & 157.43 & 159.26 & 161.33 & 157.02 & 172.71 & 157.00 & \\
\hline Water & & 256.78 & 198.64 & 252.37 & 222.05 & 260.48 & 250.89 & 342.12 & 253.79 & 157.00 \\
\hline
\end{tabular}

PVP: Polyvinylpyrrolidone, MCC: Microcrystalline cellulose, HPMC: Hydroxypropyl-methylcellulose, SA: Stearic acid, PEG: Polyethylene glycol. 
Table 5

The conservative force parameters $a_{i j}$ and $a_{i i}$ obtained by using Travis et al. [24] Eqs. (16) and (18).

\begin{tabular}{|c|c|c|c|c|c|c|c|c|c|c|}
\hline$a_{i j}$ & & PVP & MCC & HL & $\mathrm{HO}$ & $\mathrm{HC}$ & SA1 & SA2 & PEG1 & Water \\
\hline PVP & & 35.56 & & & & & & & & \\
\hline MCC & & 59.88 & 71.59 & & & & & & & \\
\hline \multirow[t]{3}{*}{ HPMC } & $\mathrm{HL}$ & 35.40 & 59.85 & 35.24 & & & & & & \\
\hline & $\mathrm{HO}$ & 46.56 & 63.97 & 46.47 & 53.77 & & & & & \\
\hline & $\mathrm{HC}$ & 30.75 & 60.77 & 30.53 & 44.88 & 23.37 & & & & \\
\hline \multirow[t]{2}{*}{ SA } & SA1 & 37.63 & 60.33 & 37.49 & 47.75 & 33.56 & 39.50 & & & \\
\hline & SA2 & 29.68 & 64.45 & 29.41 & 46.41 & 20.16 & 33.11 & 15.17 & & \\
\hline PEG1 & & 38.20 & 60.49 & 38.07 & 48.09 & 34.32 & 40.02 & 34.02 & 40.52 & \\
\hline Water & & 145.84 & 135.8 & 146.05 & 138.03 & 156.53 & 143.49 & 168.38 & 142.96 & 162.79 \\
\hline
\end{tabular}

PVP: Polyvinylpyrrolidone, MCC: Microcrystalline cellulose, HPMC: Hydroxypropyl-methylcellulose, SA: Stearic acid, PEG: Polyethylene glycol.

averaged Hildebrand solubility parameters for each repeating unit and molecules, as well as their standard deviations (see Table 1).

In Table 1, results obtained by molecular simulation are close to those obtained by HSPiP calculations. Molecular simulation results will be used next as input parameters in the DPD simulations. Table 2 shows the beads volume calculated by dividing the molecular weight $M_{w}$ by the density $e$. As anticipated, the beads volume and radius are close.

\subsubsection{DPD simulation details}

3.2.3.1. DPD parameters. The number of beads used to describe each polymer in the simulations is determined by the DPD number $n_{D P D}$ which is calculated using Eq. (20). The ratio characteristic is computed using Material Studio Synthia module [30]. The results are shown in Table 3.

Following Groot and Warren's [13] approach, the individual selfrepulsive interaction parameters $a_{i i}$ determined using Eq. (12) is equal to 157 when $N_{m}=6$. According to the authors in ref. [13], it is the same for all beads. The conservative force parameters $a_{i j}$ between every couple of beads is then calculated using the relationship (13). The results are summarized in Table 4 . We also calculate $a_{i j}$ and $a_{i i}$ using Eqs. (16) and (18) proposed by Travis et al. [24], the results are summarized in Table 5.

The harmonic spring constant of the polymer chain was set equal to 4.0 DPD reduced units (i.e. $150 \mathrm{~J} \cdot \mathrm{mol}^{-1} \cdot \AA^{-2}$ ), which is enough to keep the adjacent beads connected together along the polymer backbone [13]. Having set the coarse-graining number $N_{m}$ to 6 and the DPD number density $\bar{\rho}$ to 3 , the cut-off radius $r_{c}$ is computed from Eq. (9). We obtain $r_{c}=8.14 \AA$. The dissipation parameter $\partial$ is equal to 4.5 DPD units (i.e. $0.09043 \mathrm{~g} \cdot \mathrm{mol}^{-1} \cdot \mathrm{fs}^{-1}$ ), which is the recommended value proposed by Groot and Warren to ensure a stable simulation [13].

3.2.3.2. DPD computational details. All DPD simulations were performed within Materials Studio 7 software package (Biovia [25]). A $30 \times 30 \times 30 r_{c}^{3}$ (i.e. $24.4 \times 24.4 \times 24.4 \mathrm{~nm}$ ) simulation cell box was

Table 6

Interfacial energy results obtained by DPD simulations and compared with experimental values.

\begin{tabular}{|c|c|c|c|c|}
\hline \multirow[t]{2}{*}{ Compounds } & \multirow{2}{*}{$\begin{array}{l}\text { Number } \\
\text { density } \bar{\rho}\end{array}$} & \multicolumn{3}{|c|}{ Interfacial energy $\gamma\left(\mathrm{mJ} \cdot \mathrm{m}^{-2}\right)$} \\
\hline & & $\begin{array}{l}\text { DPD } \\
\text { Groot and } \\
\text { Warren [13] }\end{array}$ & $\begin{array}{l}\text { DPD } \\
\text { Travis et al. } \\
{[24]}\end{array}$ & Exp. from literature \\
\hline PVP & 2.98 & $48.96 \pm 0.36$ & $47.88 \pm 0.82$ & $46.7[3], 53.6[32]$ \\
\hline MCC & 4.04 & $53.19 \pm 0.49$ & $42.53 \pm 0.54$ & $53.1[3]$ \\
\hline HPMC & 3.09 & $43.40 \pm 1.16$ & $33.76 \pm 0.22$ & $\begin{array}{l}34[2], 38.4[3], 43.1 \\
{[33], 48.4[34]}\end{array}$ \\
\hline SA & 2.26 & $31.38 \pm 0.67$ & $19.75 \pm 0.28$ & $28.9[35]$ \\
\hline PEG400 & 2.98 & $54.14 \pm 0.37$ & $43.52 \pm 0.52$ & $46.7[36]$ \\
\hline
\end{tabular}

PVP: Polyvinylpyrrolidone, MCC: Microcrystalline cellulose, HPMC: Hydroxypropyl-methylcellulose, SA: Stearic acid, PEG: Polyethylene glycol. adopted and periodic boundary conditions were applied in all three directions. Initially, the beads were randomly dispersed in the simulation cell. Each DPD simulation ran for 1000 DPD units (i.e. 5374.17 ps), which was sufficient to get a steady phase. The integration time was taken as $\mathrm{t}=0.02 \mathrm{DPD}$ units (i.e. $107.48 \mathrm{fs}$ ). DPD simulations were run in the canonical thermodynamic NVT ensemble at a temperature of $\mathrm{T}=298 \mathrm{~K}$

We evaluated the interfacial energy $\gamma$ by dividing a $30 \times 6 \times 6$ $r_{c}^{3}$ simulation box into a number of $\mathrm{x}$-normal slabs. This way, the tensor elements will be a function of the distance in the $x$ direction [25]. An equilibration period of 1400 DPD units (i.e. 7526.59 ps) steps was used and followed by a production run of 600 DPD units (i.e. $3225.69 \mathrm{ps}$ ). In the DPD method, the masses of all particles are normally chosen to be the same and equal to 108 amu for 6 water molecules in one bead. Therefore, for the interfacial energy calculations, we matched the mass of the beads of each compound to that value (108 amu). This was done by multiplying the simulation target number density $\bar{\rho}$ by the relative density $e$ obtained by averaging the weight density of the beads (see Table 1) of each compound. Interfacial energy $\gamma$ in the DPD simulations can then be calculated using the Irving-Kirkwood [31] equation by integrating the difference between normal and tangential stresses across the interface.

$\bar{\gamma}=\int\left(<\bar{P}_{x x}>-0.5\left(<\bar{P}_{y y}>+<\bar{P}_{z z}>\right) \mathrm{d} \bar{x}\right.$

$\gamma=\bar{\gamma} \frac{k_{B} T}{r_{c}^{2}}$

where $\bar{x}=x / r_{c}$ and $P$ is the pressure tensor that consists of three diagonal components $P_{x x}, P_{y y}$, and $P_{z z}$.

\subsection{Experimental methods}

\subsubsection{Preparation of the suspensions}

HPMC-SA mixture was prepared by adding the cellulose polymer in deionized water previously heated to $80{ }^{\circ} \mathrm{C}$. The mixture was then homogenized by moderate agitation for 30 to 60 min using a rotor stator homogenizer (Ultraturrax T25, Janke and Kunkel, Germany) at $85^{\circ} \mathrm{C}$. Stearic acid was then added to the HPMC solution progressively under agitation until it was evenly dispersed. The mixture was then cooled using an ice bath under agitation for $30 \mathrm{~min}$. Solutions were thereafter degassed at 50 mbar for $2 \mathrm{~h}$. To attain maximum stabilization, the readily prepared solutions were stored immediately at $5{ }^{\circ} \mathrm{C}$ for at least $24 \mathrm{~h}$.

The same protocol was used for the preparation of PVP-SA mixture.

\subsubsection{The Cryo-SEM technique}

Morphological examination of the structure of the mixtures was carried out using the Cryo-SEM technique. First, in order to fix the structure and the morphology of the samples, rapid freezing of the sample in pasty nitrogen $\left(-210^{\circ} \mathrm{C}\right)$ or in liquid ethane $\left(-172{ }^{\circ} \mathrm{C}\right)$ was used. 


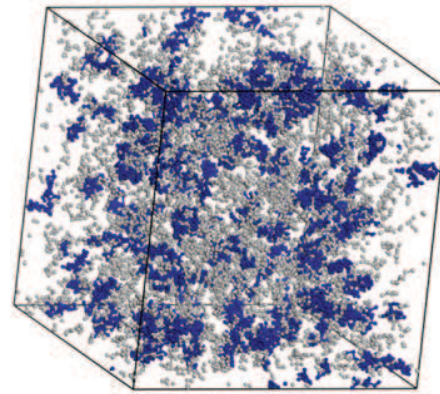

(a) Time: $537 \mathrm{ps}$

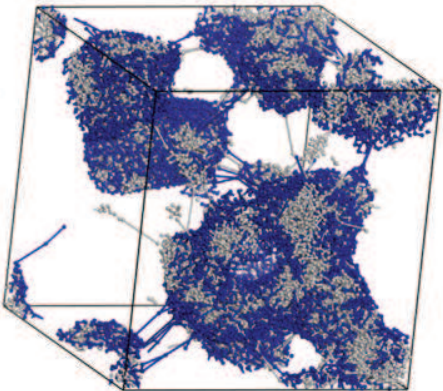

(b) Time: $1074 \mathrm{ps}$

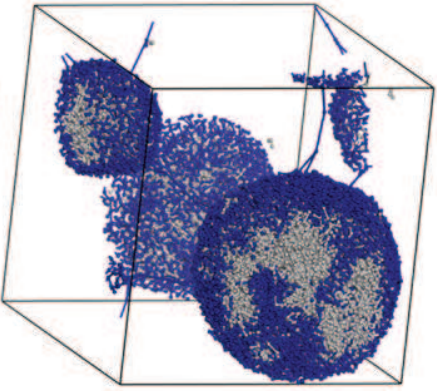

(c) Time: $5374 \mathrm{ps}$

HPMC OSA

Fig. 4. DPD simulation of HPMC (Hydroxypropyl-methylcellulose, blue, 10\%)-SA (Stearic acid, grey, 10\%) mixture in water (transparent, 80\%).

The frozen samples were then fractured by striking them with a cold scalpel. The revealed fractured surface was then metal-coated with a beam of electrons and introduced in the analysis chamber to be examined in a scanning electron microscope (SEM) (Hitachi MEB ESEM Quanta $250 \mathrm{FEG}$ FEI) while being maintained at $-135^{\circ} \mathrm{C}$. In a second phase of the analysis, the fractured samples were sublimated at $-90{ }^{\circ} \mathrm{C}$ for 20 to $40 \mathrm{~min}$ in a vacuum SEM cool chamber before examination in the cryo-stage SEM. The sublimation step was performed to remove water from frozen samples and to expose the first layer of particles inside the dispersion.

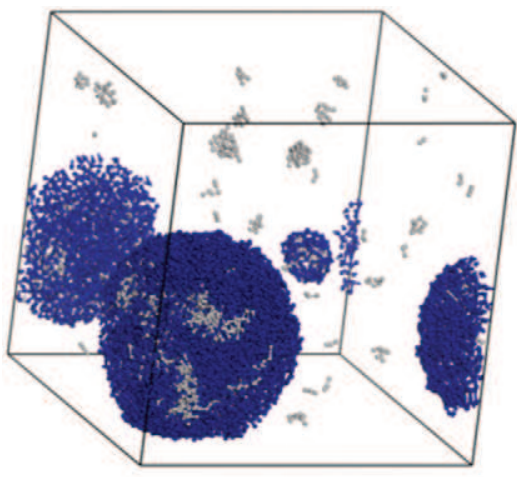

(a) HPMC-SA $10 \%-2 \%(w / w)$ $30 \times 30 \times 30 r c^{3}$ box size

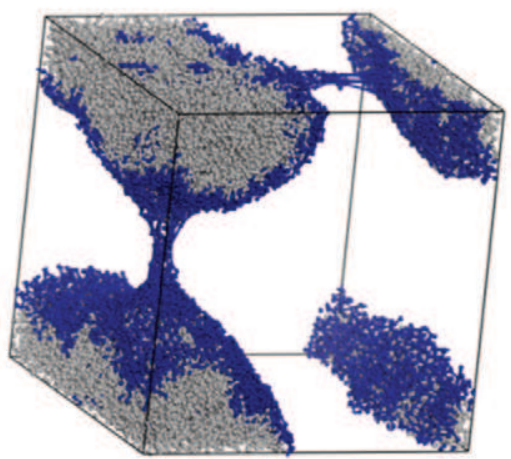

(c) HPMC-SA $10 \%-20 \%(\mathrm{w} / \mathrm{w})$ $30 \times 30 \times 30 r c^{3}$ box size

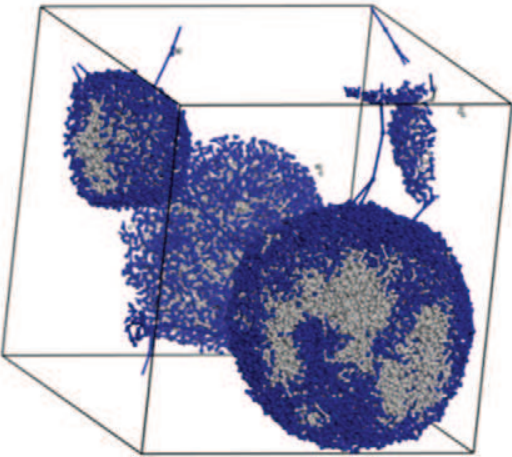

(b) HPMC-SA $10 \%-10 \%(w / w)$ $30 \times 30 \times 30 r^{3}$ box size

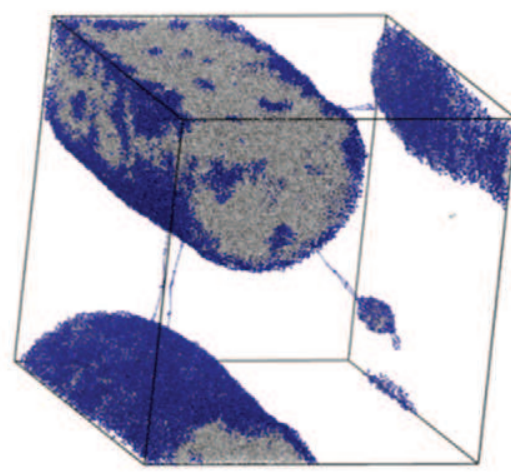

(d) HPMC-SA $10 \%-20 \%(w / w)$ $60 \times 60 \times 60 r e^{3}$ box size 

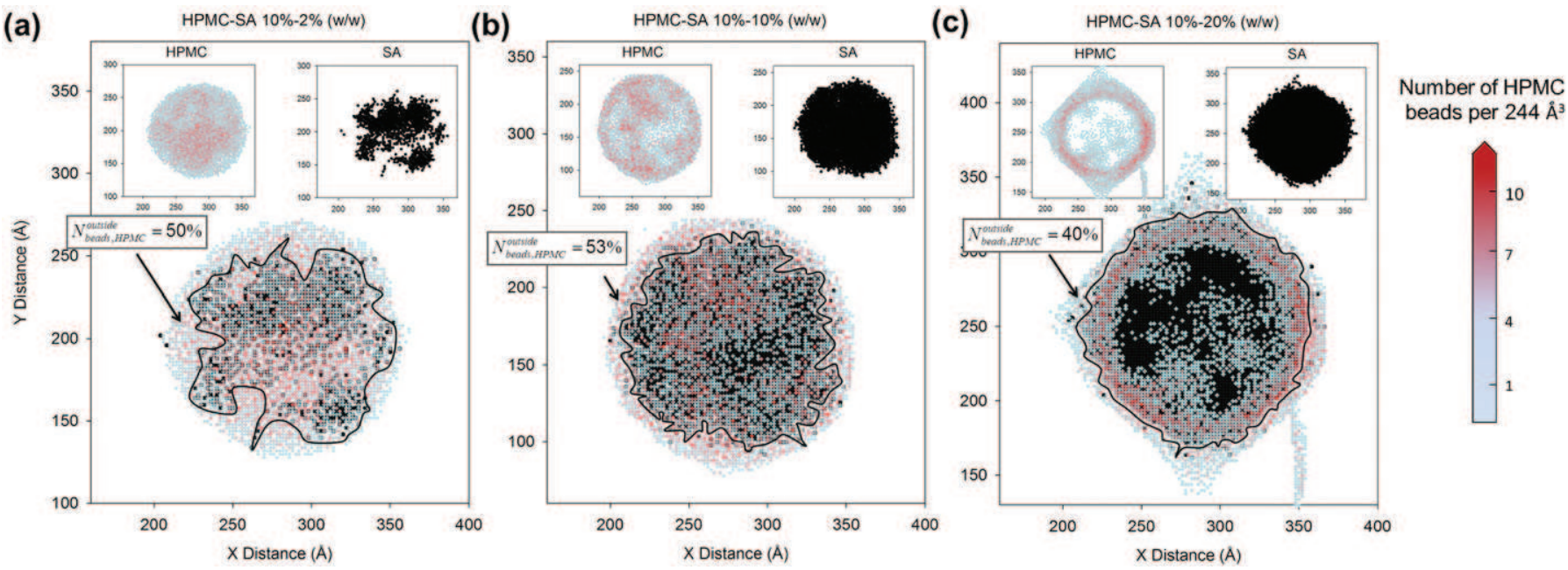

Fig. 6. Distribution of HPMC beads around and through SA agglomerate under different amounts of SA, HPMC: Hydroxypropyl-methylcellulose, SA: Stearic acid.

\section{Results and discussion}

\subsection{DPD simulations results}

When preparing a binder or coating solution, the challenge is to fabricate a polymeric solution with a high hydrophobic SA content while maintaining the stability of the suspension. To study the stability of HPMC-SA coating solution under different percentages of SA, we use DPD simulations. Then, we investigate the influence of polymer nature (HPMC, PVP, MCC) on the SA based coatings.

\subsubsection{Interfacial energy}

Before using the DPD method on ternary aqueous systems, we calculate interfacial energies of the compounds. We used the DPD method proposed by Groot and Warren [13] and the DPD method proposed by Travis et al. [24]. Then, we compared the results with experimental values from literature. Computed interfacial energy values are presented in Table 6.

Interfacial energy values obtained following Groot and Warren's [13] method and Travis et al. [24] method, are calculated using the conservative force parameters previously presented in Table 4 and in Table 5 respectively.

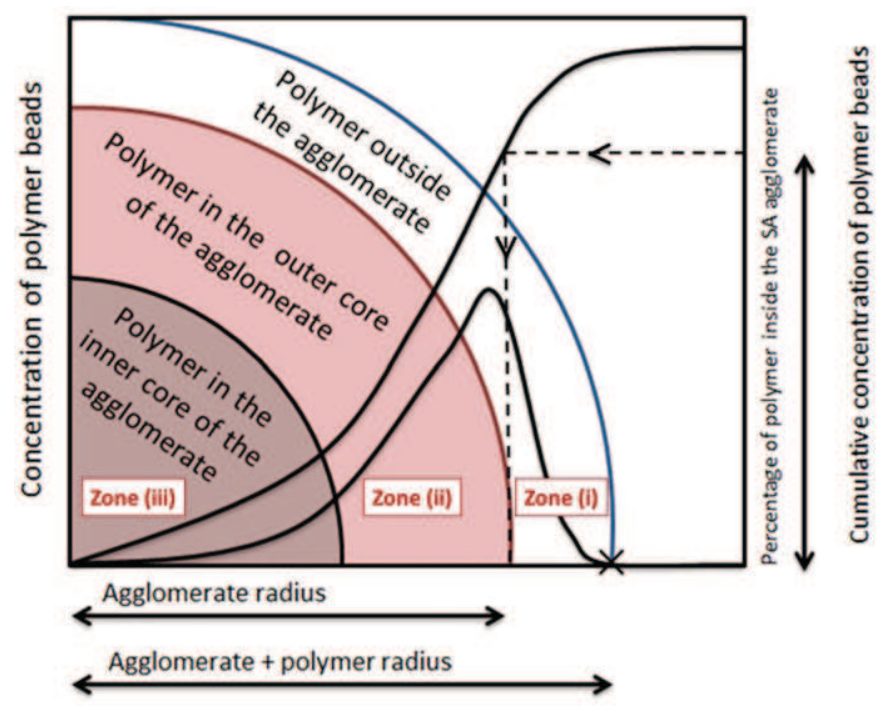

Fig. 7. Schematic representation of the distribution of polymer in relation to the agglomerate structure and size.
Interfacial energy values obtained by DPD simulations following Groot and Warren [13] and Travis et al. [24] are close to the experimental values, but Groot and Warren's approach give closer values. Henceforth, we will adopt the DPD equations of Groot and Warren. In the remaining of the study, we will use Groot and Warren's [13] density value; $\bar{\rho}=3$ since we are only interested in the structures of the agglomerates in the dispersions.

\subsubsection{Influence of SA concentration on HPMC-SA agglomerate in water}

Fig. 4 shows three snapshots of configurations of HPMC-SA $(10 \%-10 \%(w / w))$ in water (transparent) after 537.41 ps (Fig. $4(a))$, 1074.83 ps (Fig. 4(b)) and 5374.17 ps (Fig. 4(c)) of simulation time. Initially HPMC and SA beads are randomly dispersed in water (Fig. 4(a)). Hydrophobic SA molecules progressively agglomerate under the action of the repulsion forces of the water beads (Fig. 4(b)). At the same time, HPMC beads gradually diffuse through water and redistribute on the outer surface of the SA agglomerate. As the simulation progresses, the HPMC-SA agglomerate increases in size until the HPMC matrix completely surrounds SA through polymer entanglement and form a thick layer between SA and water (Fig. 4(c)).

In Fig. 5, we present structures of HPMC-SA mixture under different fractions of SA. All the images show the last step of the DPD simulation when the equilibrium state is reached. When SA fraction is $2 \%(\mathrm{w} / \mathrm{w})$ (Fig. 5 (a)), HPMC polymer completely covers a large SA agglomerate. We notice that some small SA agglomerates move freely in the simulation cell. Upon increasing the SA percentage, we observe a growth of SA agglomerates and a decrease of the number of loosen SA agglomerates. When the SA weight percentage is up to $20 \%(\mathrm{w} / \mathrm{w})$, the agglomerating structure of HPMC-SA is not spherical anymore and a tubular structure is formed (Fig. 5(c)). To waive a possible artifact due to the box size, we display a simulation with an 8 times larger box in Fig. 5(d). Again, a tubular structure is obtained, with a bigger radius. Moreover, there is no loose SA agglomerate in the water (Fig. 5(c) and (d)). We also notice that some HPMC penetrate the inner core of the SA agglomerate to various extend depending on the amount of SA.

An important requirement to prevent agglomeration is that the stabilizing agent has to be adsorbed strongly enough on the surface of the particle. If the polymer is only weakly adsorbed, then, it is possible that desorption can take place even during Brownian collisions (without deliberately shearing the system). Thus, agglomeration may take place within the system on standing [37]. Spontaneous, weak, slow agglomeration can also occur in systems where the adsorption is strong, but where the adsorbed layer is thin [37] and may results in inhomogeneous polymeric coating film. The strength of the adsorption in our 


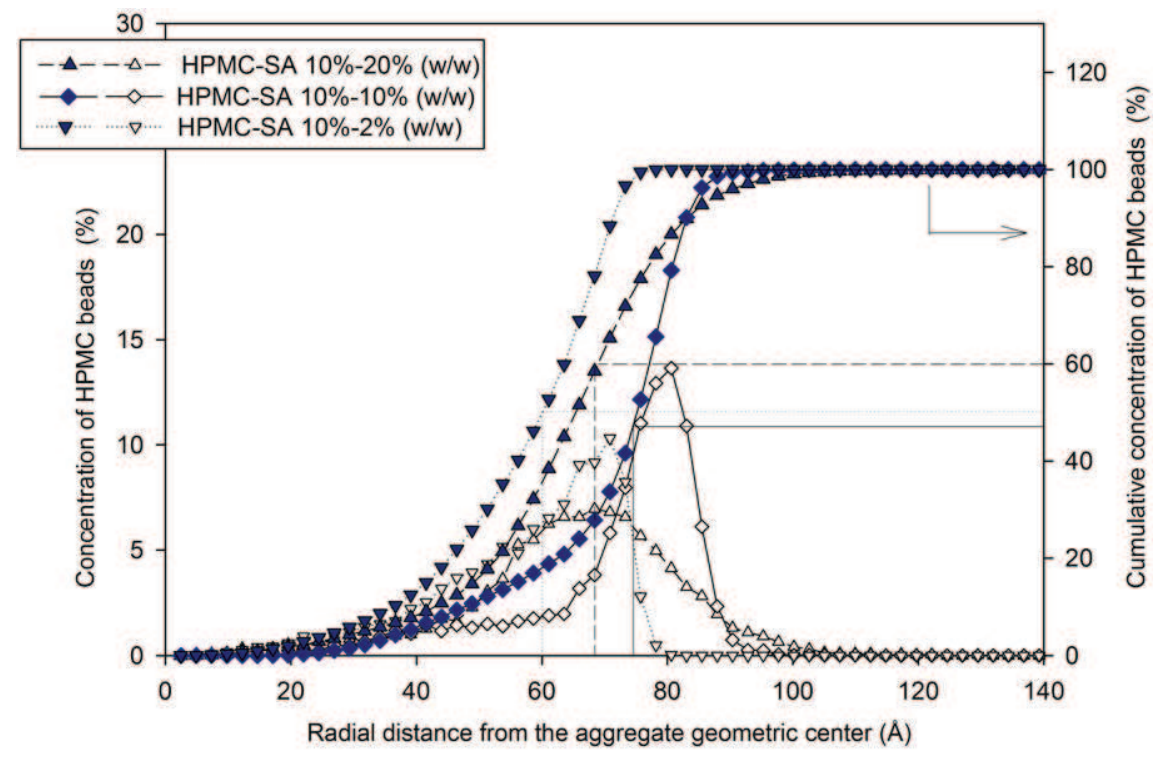

Fig. 8. Concentration of HPMC beads as a function of the radial distance from SA agglomerate geometric origin. HPMC: Hydroxypropyl-methylcellulose and SA: Stearic acid.

DPD simulations can be assessed by the amount of stabilizing agent beads which are inside the agglomerate.

Fig. 6 shows the distribution of HPMC around and through SA agglomerate as the percentage of SA increases. The percentage of beads of polymer that cover SA agglomerate $N_{\text {beads,polymer }}^{\text {outsin }}$ can be calculated by using the following equation:

$$
\begin{aligned}
& N_{\text {beads.polymer }}^{\text {outside }} \\
& \quad=\frac{100}{N_{\text {Beads,polymer }}}\left(N_{\text {Beads.polymer }}-\sum_{i} \Delta\left(\prod_{j} 1-\Delta\left(\left|r_{0}-r_{i}\right|+\left|r_{i}-r_{j}\right|-\left|r_{0}-r_{j}\right| \pm 2 r_{\text {water }}\right)\right)\right) .
\end{aligned}
$$

Where $r_{i}$ denotes the position vector of the $i$ th polymer bead, $i=1 \ldots N_{\text {Beads,polymer. }} N_{\text {Beads, polymer }}$ is the total number of polymer beads (HPMC, PVP or MCC in our case), $r_{j}$ denotes the position vector of the $j$ th SA bead, $r_{o}$ is the position vector of the bead at the geometric center of the HPMC-SA agglomerate, $r_{\text {water }}$ is the radius of a water bead which is roughly equal to the radius of the other beads (see Table 2 ), and $\Delta$ is the Dirac function.

In Fig. 6(a), the agglomerate structure with $2 \% \mathrm{SA}(\mathrm{w} / \mathrm{w})$ is like an assembly of small patches of SA embedded in a matrix of HPMC that globally forms spherical agglomerate. The percentage of HPMC beads outside all SA beads is equal to $50 \%$.

In Fig. 6(b), SA beads form a large spherical cluster surrounded by $N_{\text {beads, }}^{\text {outsidymer }}=53 \%$ of HPMC. Therefore $47 \%$ of HPMC beads have diffused inside the SA inner core. The colored HPMC density scale indicates that HPMC is well distributed inside the SA inner core.

In Fig. 5(c) and (d), the increase of the SA concentration to $20 \%$ $(\mathrm{w} / \mathrm{w})$ for the same HMPC content changed the stable structure of the system. From a spherical shape of SA cluster obtained for lower SA concentration at 2 and $10 \%(w / w)$, we ended up with a tubular shape at $20 \%$ $(\mathrm{w} / \mathrm{w})$ of SA. Laboulfie et al. [38] also noticed in their experiments that an increase of SA concentration in HPMC-water solution destabilized the suspension and favored the formation of large SA agglomerate. From our simulations in Fig. 6(c), with the increase of SA beads, we notice that the density of HPMC beads that covers the SA cluster decreases to $N_{\text {beads, }}^{\text {outsolymer }}=40 \%$. We also observe a denser SA core and that less HPMC molecules are able to diffuse deeply in the SA core. This hints that HPMC polymer would be less likely to get through the SA agglomerate as SA concentration reaches $20 \%(\mathrm{w} / \mathrm{w})$. In summary, at large SA concentration, the SA molecules tend to cluster together and push at the fringes the HPMC molecules.

The distribution of the polymer in the agglomerate can be subdivided into three zones; i) polymer outside the agglomerate, ii) polymer in the outer core of the agglomerates, and iii) polymer in the inner core of the agglomerate (see Fig. 7).

The polymer nature and distribution in the agglomerate affect the flexibility of the coating film and the mechanical strength of the final granule. For example, a homogenous distribution of the polymer inside the colloidal agglomerate (i.e. the polymer is well dispersed in

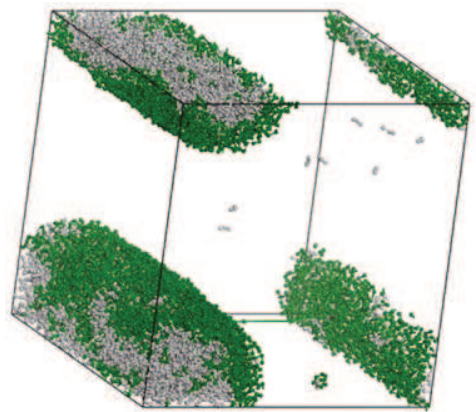

a) $\mathrm{PVPO}-\mathrm{SAO}$

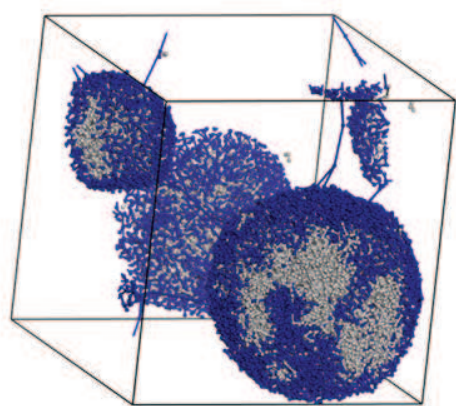

b) HPMC -SAO

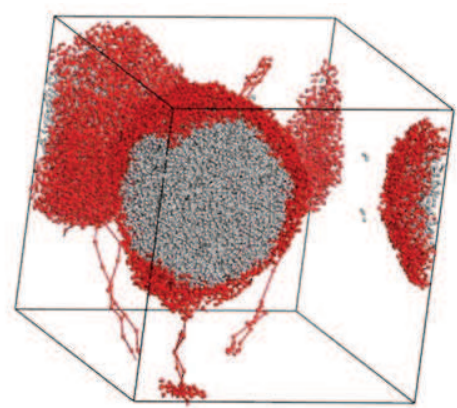

c) $\mathrm{MCCO}-\mathrm{SA}$

Fig. 9. Snapshots of DPD simulation of PVP-SA, HPMC-SA and MCC-SA in water 10\%-10\% (w/w) when equilibrium state is reached. PVP: Polyvinylpyrrolidone, MCC: Microcrystalline cellulose, HPMC: Hydroxypropyl-methylcellulose, SA: Stearic acid. 


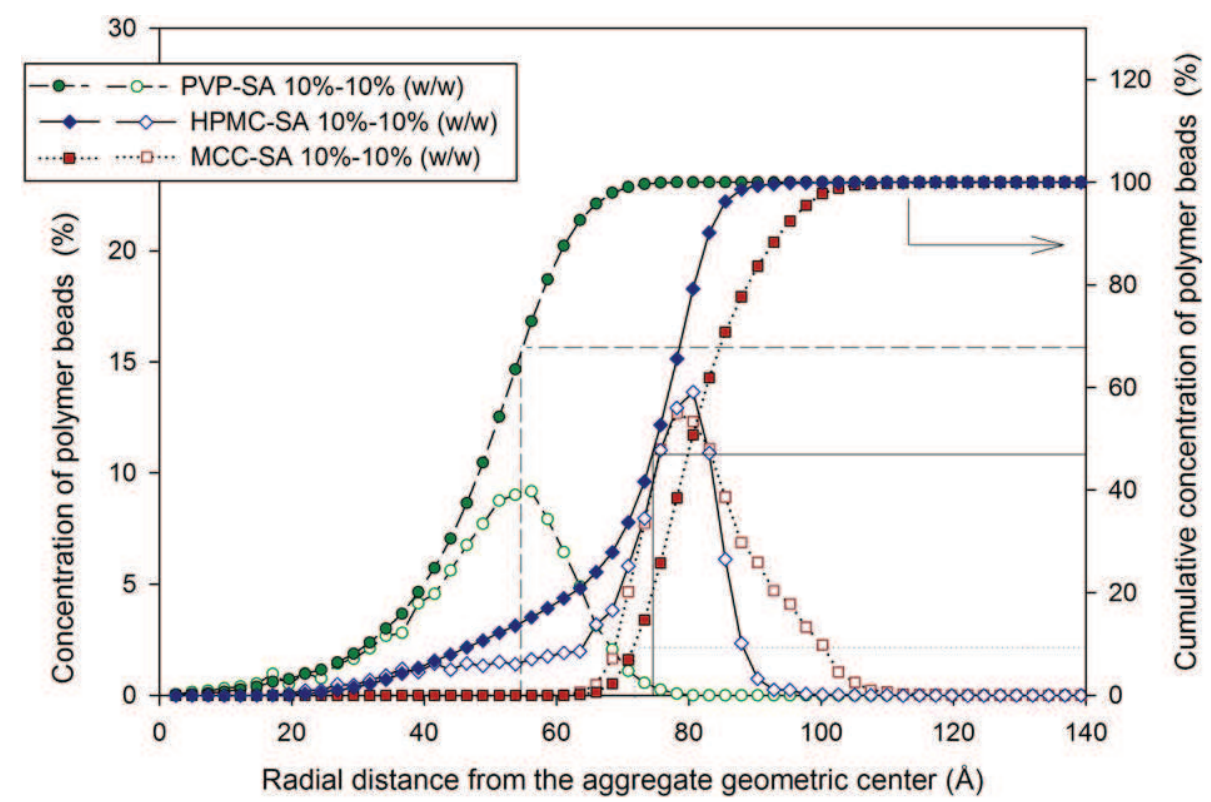

Fig. 10. Concentration of polymer beads as a function of radial distance from polymer-SA agglomerate geometric center. PVP: Polyvinylpyrrolidone, MCC: Microcrystalline cellulose, HPMC: Hydroxypropyl-methylcellulose and SA: Stearic acid.

zone (ii) and (iii) (see Fig. 7)) improves the plastic behavior of the coting film. Also, high polymer content in the inner core of the agglomerate (see Fig. 7) may increase the strength of the final granule. Müller et al. [39] investigated this effect and considered the influence of the binder content on the strength of the agglomerate product.

Fig. 8 shows a distribution function $\Gamma(r, d r)$ that represents the percentage of beads of HPMC as a function of the radial distance starting from the HPMC-SA agglomerate geometric center. The cumulative concentration is also showed. $\Gamma(r, d r)$ gives insights about the uniformity of the HPMC polymer distribution inside SA agglomerate and the size of the SA agglomerate (see also Fig. 7).
$\Gamma(r, d r)$ is obtained by applying the following equation:

$\Gamma(r, d r)=\frac{100}{N_{\text {Beads,polymer }}} \sum_{i} \Delta\left(H\left(\left|r-r_{o}\right|-\left|r_{i}-r_{o}\right|\right)+H\left(\left|r_{i}-r_{o}\right|-\left|r+d r-r_{o}\right|\right)\right)$

where $H$ is the Heaviside function.

The higher the distribution curve $\Gamma(r, d r)$ peak at a given radial distance, the more polymer beads are at that distance. The narrower $\Gamma(r, d r)$, the denser the polymer shell outside the SA agglomerate. The vertical lines in Fig. 8 separate the percentage of HPMC that are inside (a)

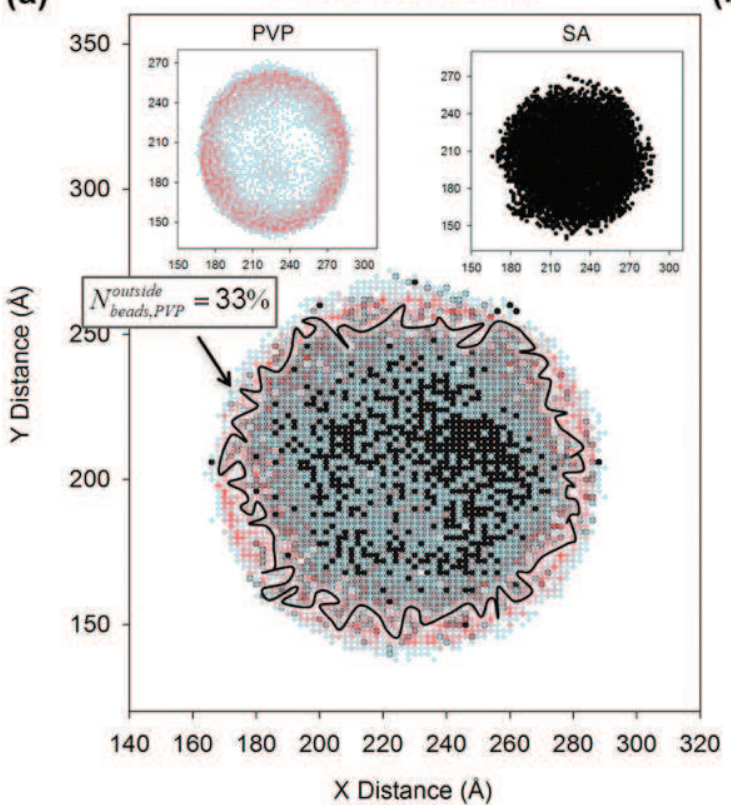

(b)

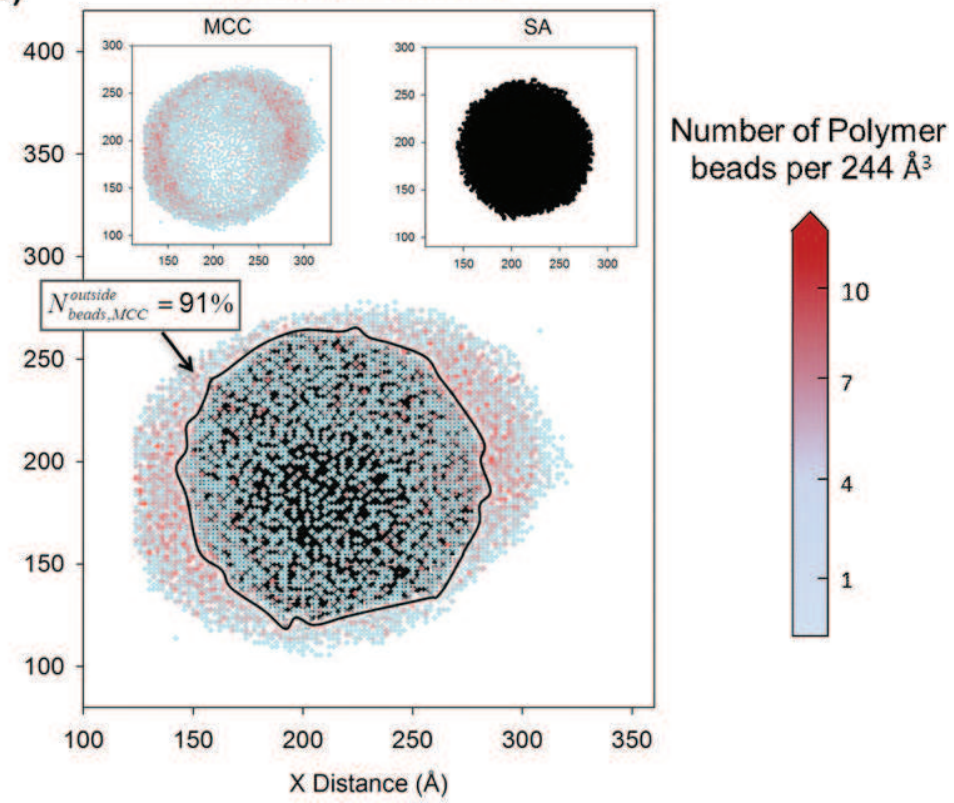

Fig. 11. Distribution of polymer beads (PVP and MCC) around and through SA agglomerate. PVP: Polyvinylpyrrolidone, MCC: Microcrystalline cellulose and SA: Stearic acid. 


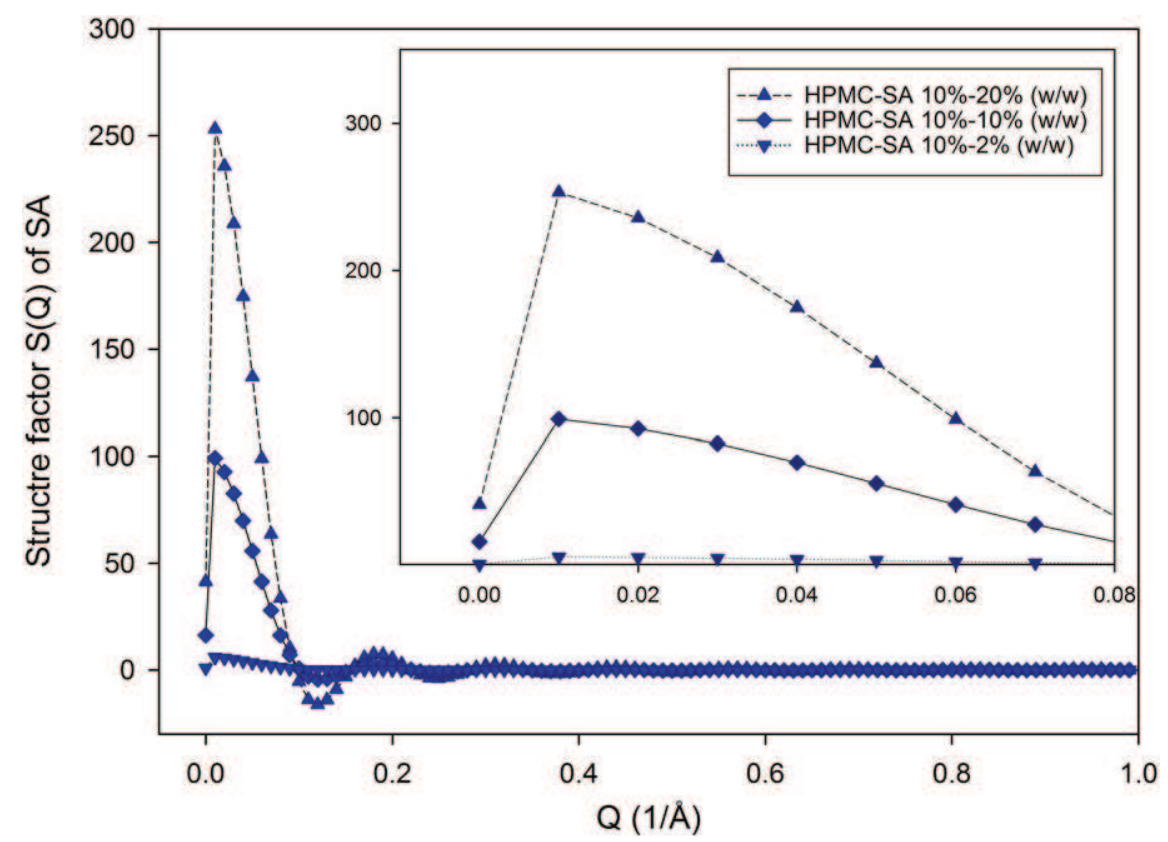

Fig. 12. Average structure factor S(Q) of HPMC-SA under different percentages of SA. HPMC: Hydroxypropyl-methylcellulose, SA: Stearic acid.

the SA agglomerate from the outside ones. The percentages at which the vertical lines are drawn are taken from $N_{\text {beads,polymer }}^{\text {outside }}$ values calculated before using Eq. (23). They enable us to estimate an equivalent SA core radius $R_{\text {equivalent }}$ given by the intersection between the cumulative distribution curve and the corresponding vertical line (see Figs. 7 and 8 ).

From Fig. 8, we obtain $R_{\text {equivalent }}^{\text {sphere }}=60 \AA$ for $2 \%(\mathrm{w} / \mathrm{w})$ of $\mathrm{SA}$, $R_{\text {equivalent }}^{\text {sphere }}=74 \AA$ for $10 \%(\mathrm{w} / \mathrm{w})$ of $\mathrm{SA}$, and $R_{\text {equivalent }}^{\text {tubular }}=69 \AA$ for the tubular structure obtained for $20 \%(w / w)$ of SA.

In Fig. 8, HPMC-SA 10\%-2\% (w/w) displays a wider spread distribution than HPMC-SA $10 \%-10 \%(\mathrm{w} / \mathrm{w})$ indicating that more HPMC beads have diffused inside the SA agglomerate at $2 \%$ of SA. This confirms the conclusions obtained from Fig. 6 (a) and (b). HPMC-SA 10\%-2\% (w/w) shows lower peak and lower $R_{\text {equivalent }}^{\text {sphere }}$ than HPMC-SA $10 \%-10 \%(\mathrm{w} / \mathrm{w})$ because there is only $2 \%(\mathrm{w} / \mathrm{w})$ of SA interacting with $10 \%(\mathrm{w} / \mathrm{w})$ of HPMC.

Both HPMC-SA 10\%-2\% (w/w) and HPMC-SA 10\%-10\% (w/w) show a sharp distribution that peaks at a radial distance greater than the corresponding $R_{\text {equivalent }}^{\text {sphere }}$ value for the SA inner core. This means that high percentage of HPMC beads are distributed on the outer surface of SA cluster. This is the best case scenario where there are enough HPMC beads inside SA agglomerate to hold the SA agglomerate in position, and enough of them outside to cover the SA agglomerate.

As the percentage of SA increases to $20 \%(\mathrm{w} / \mathrm{w})$, the peak shifts to a lower value below the corresponding $R_{\text {equivalent. }}^{\text {sphere }}$ This is correlated with the increase of HPMC percentage beads inside SA agglomerate to $1-N_{\text {beads, }}^{\text {outsidymer }}=60 \%$. Additionally, the increasing number of beads

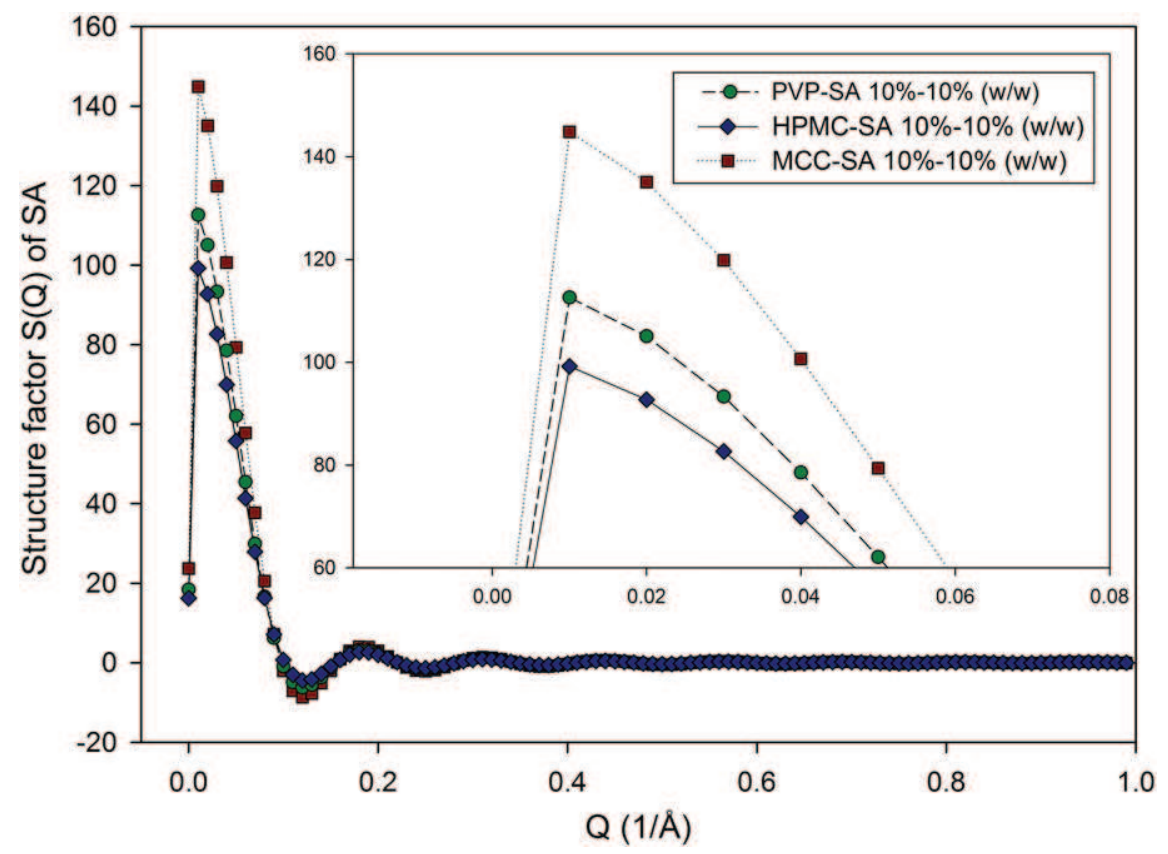

Fig. 13. Average structure factor S(Q) of stearic acid (SA) agglomerates formed when using different polymeric compounds, PVP: Polyvinylpyrrolidone, MCC: Microcrystalline cellulose, HPMC: Hydroxypropyl-methylcellulose. 


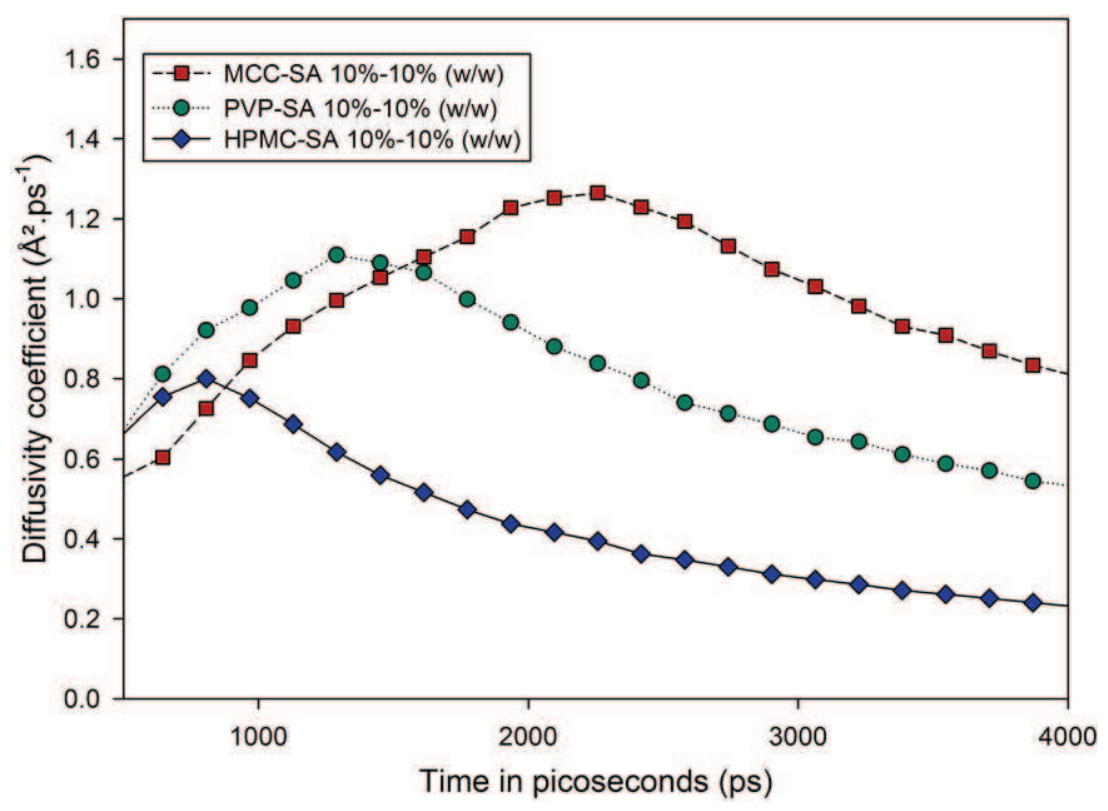

Fig. 14. Evolution of the diffusivity of HPMC, PVP and MCC in the mixtures HPMC-AS, PVP-AS and MCC-AS (10\%-10\% (w/w) in water) respectively, as a function of time in DPD units. HPMC: Hydroxypropyl-methylcellulose, PVP: Polyvinylpyrrolidone, MCC: Microcrystalline cellulose, SA: Stearic acid.

of HPMC inside the SA matrix makes the layer that covers SA agglomerate less thick.

From these three simulations at various SA concentrations for the same HPMC content, we may infer that HPMC can stabilize an aqueous SA suspension provided that the proportion of HMPC vs. SA is high enough and that a network of HPMC molecules is diffused in depth in the SA core. This was achieved for the $2 \%$ and $10 \%(\mathrm{w} / \mathrm{w}$ ) of SA cases. At higher SA load, the tubular structure of SA, with a lower surface area than the spherical structure becomes energetically more favorable.

\subsubsection{Influence of the polymer nature on the SA based coating}

To study the behavior of each polymer in the presence of SA hydrophobic filler in aqueous systems, we ran DPD simulations of polymer-SA $10 \%-10 \%(\mathrm{w} / \mathrm{w})$ where the polymers are PVP, HPMC and MCC.

Fig. 9 shows the final structure of the different mixtures (HPMC-SA, PVP-SA and MCC-SA in water) when equilibrium state is reached. Regarding the PVP-SA mixture (Fig. 9(a)), PVP polymer tends to surround SA molecules in an aqueous environment. However, a tubular structure is obtained unlike the spherical structure in the case of HPMC-SA 10\%-10\% (w/w) blend (Fig. 9(b)). Fig. 10 shows the percentage of beads of each polymer as a function of the radial distance starting from the SA agglomerate geometric origin. PVP polymers diffuse in the SA agglomerate as shown in Fig. 10 by the curve of concentration of PVP polymer that is broader than that of HPMC and has a lower peak than the other curves.

Fig. 11 shows the distribution of polymer beads (PVP and MCC) around and through SA. We have also computed $N_{\text {beads, }}^{\text {outsidymer }}$ by using Eq. (23). As shown in Fig. 11 (a) the percentage of beads of PVP inside

Table 7

Affinity predicted with PVP, MCC and HPMC in water using different approaches.

\begin{tabular}{|c|c|c|c|c|c|c|c|c|c|}
\hline \multirow[t]{3}{*}{ A } & \multicolumn{9}{|l|}{ B } \\
\hline & \multicolumn{3}{|l|}{ PVP } & \multicolumn{3}{|l|}{ MCC } & \multicolumn{3}{|c|}{ HPMC } \\
\hline & DPD & $\begin{array}{l}W_{\text {Adhesion }} \\
{[4]}\end{array}$ & $\begin{array}{l}\sigma_{\text {tensile }} \\
{[4]}\end{array}$ & DPD & $\begin{array}{l}W_{\text {Adhesion }} \\
{[4]}\end{array}$ & $\begin{array}{l}\sigma_{\text {tensile }} \\
{[4]}\end{array}$ & DPD & $\begin{array}{l}W_{\text {Adhesion }} \\
{[4]}\end{array}$ & $\begin{array}{l}\sigma_{\text {tensile }} \\
{[4]}\end{array}$ \\
\hline SA & $\mathrm{X}$ & 0 & 0 & $X$ & $\mathrm{X}$ & $X$ & $X$ & $X$ & $\mathrm{X}$ \\
\hline
\end{tabular}

PVP: Polyvinylpyrrolidone, MCC: Microcrystalline cellulose, HPMC: Hydroxypropyl-methylcellulose, SA: Stearic acid, O: A adhere on B, X: B adhere on A, M: A and B are mixed in water.
SA agglomerate is high, about $67 \%$, which leaves $33 \%$ that surrounds SA agglomerate, thus, the layer of PVP outside SA agglomerate is thin compared to the one formed by HPMC in the HPMC-SA 10\%-10\% $(\mathrm{w} / \mathrm{w})$ mixture. The colored PVP density scale in Fig. 11 shows that the majority of PVP beads which are inside the SA agglomerate are distributed in the outer core of SA agglomerate.

In Fig. 9 (b), MCC interposes on the surface of SA without diffusing and forms a spherical shape. The peaked MCC curve in Fig. 10 implies that MCC beads tend to gather exclusively outside SA cluster and form a thick layer. Moreover, the radius of the SA inner core $R_{\text {equivalent }}^{\text {sphere }}=71.76 \AA$ is at the bottom of the MCC distribution curve. This indicates that the layer of MCC made by the beads which are in the outer core of SA agglomerate is very thin.

In Fig. 11(b), the percentage of MCC that diffuses inside SA agglomerate is $9 \%$ (see Fig. 11(b)). MCC beads are mainly distributed in the outer area of the SA agglomerate. The amount of MCC inside SA agglomerate is significantly low compared to HPMC (Fig. 6(b)) and PVP (Fig. 11(a)). Consequently, since colloidal dispersions always show Brownian motion and hence collide with each other frequently [5], the physical bond between SA and MCC is susceptible to detach, and SA particles could escape the MCC layer, and therefore, form large agglomerate.

By comparing the previous simulation results on the effect of $10 \%$ (w/w) of PVP, HPMC and MCC on $10 \%(w / w)$ of SA, we may deduce that PVP is able to stabilize SA particle but it's not as effective as HPMC. The percentage of MCC beads which are in the core of SA agglomerate is very low compared to HPMC and PVP. This tells us that MCC may be a good dispersant but not a good stabilizer for SA.

\subsubsection{Structure factor and diffusivity coefficient}

4.1.4.1. Structure factor. The structure factor $S(Q)$ describes the distribution of scattering material in real space and thus, accounts of the degree of a particle packing structure inside a colloidal dispersion [40]. $Q$ here is the scattering vector. $S(Q)$ is derived by the Fourier transformation of the radial distribution of the DPD simulation results $g(r)$ [41]. In order to include all possible pair interactions and to increases the resolution of the spectrum, DPD analysis of the structure factor was done with a large cut-off distance equal to $314 \AA$. 


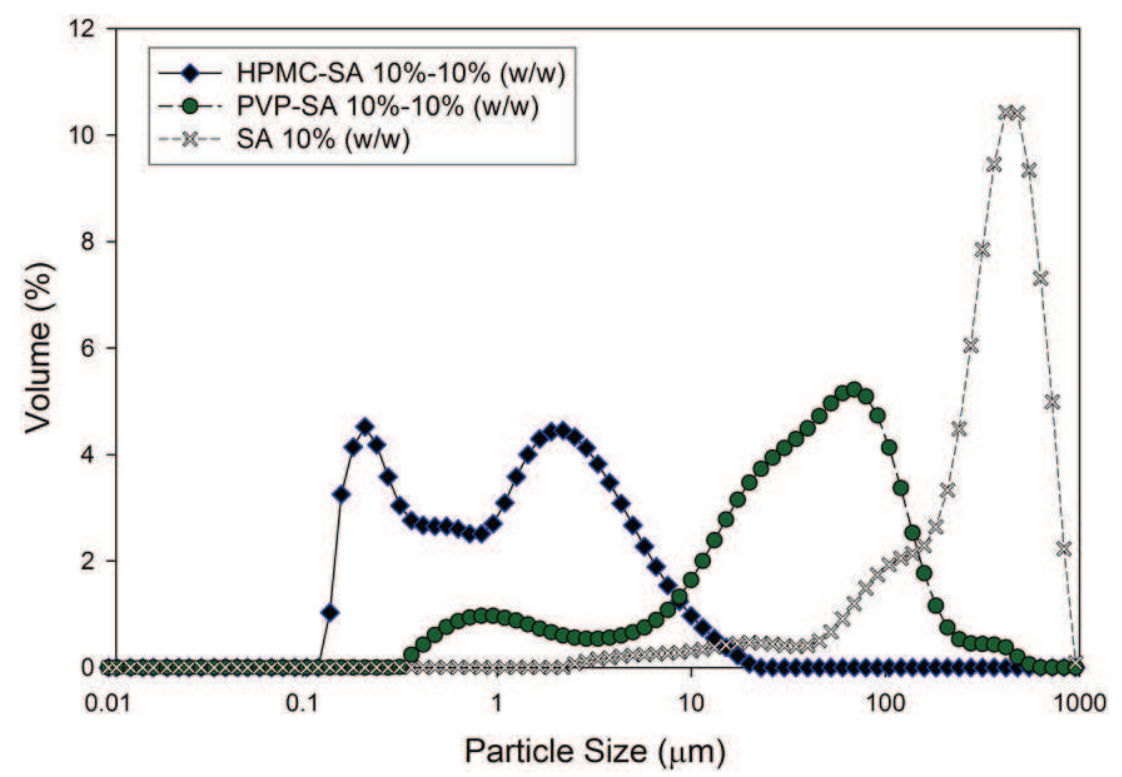

Fig. 15. Particle size distribution in volume of HPMC-SA, PVP-SA and pure SA in water. HPMC: Hydroxypropyl-methylcellulose, PVP: Polyvinylpyrrolidone, SA: Stearic acid.

Fig. 12 shows the evolution of the structure factor $S(Q)$ of SA beads in the HPMC-SA mixture when the SA fraction is increased. Each curve was averaged over the last 50 DPD time units (i.e. $268.7 \mathrm{ps)} \mathrm{of} \mathrm{simulation.} \mathrm{A}$ pronounced first peak of the structure factor translates into a higher sized agglomerate formation and more organized structure. The peak of the structure factor at $2 \%(\mathrm{w} / \mathrm{w})$ of SA is very low, thus, the SA beads are unorganized and tend to scatter (Fig. 12). This corresponds to the small patches of SA trapped inside the HPMC matrix obtained in Fig. 6(a). The curve sharpens for higher SA fractions which indicate that the SA beads are more ordered. This behaviour is reminiscent of an agglomerate size growth.

The average structure factor curves of SA in PVP-SA, HPMC-SA and MCC-SA are shown in Fig. 13. MCC-SA mixture demonstrates the highest first peak, indicating formation of large SA agglomerate limited by the neat MCC spherical shell. The SA agglomerate structure is also better organized in the case of MCC-SA than in the cases of PVP-SA and HPMC-SA.

4.1.4.2. Diffusivity coefficient. The diffusivity coefficient $D$ in DPD simulations is anticipated from Einstein's mean square displacement relation $[41,42]$. The equation that we used to calculate $D$ is given by the following formula:

$D=\frac{1}{6} \lim _{t \rightarrow \infty} \frac{d}{d t}<\left|r_{i}(t)-r_{i}(0)\right|^{2}>$

Ideally, adsorption of polymer should occur relatively quickly during the stabilization [37]. Fig. 14 shows the evolution of the coefficient of diffusivity over DPD time of HPMC, PVP and MCC when mixed with SA in aqueous environment. We notice that the diffusion coefficient

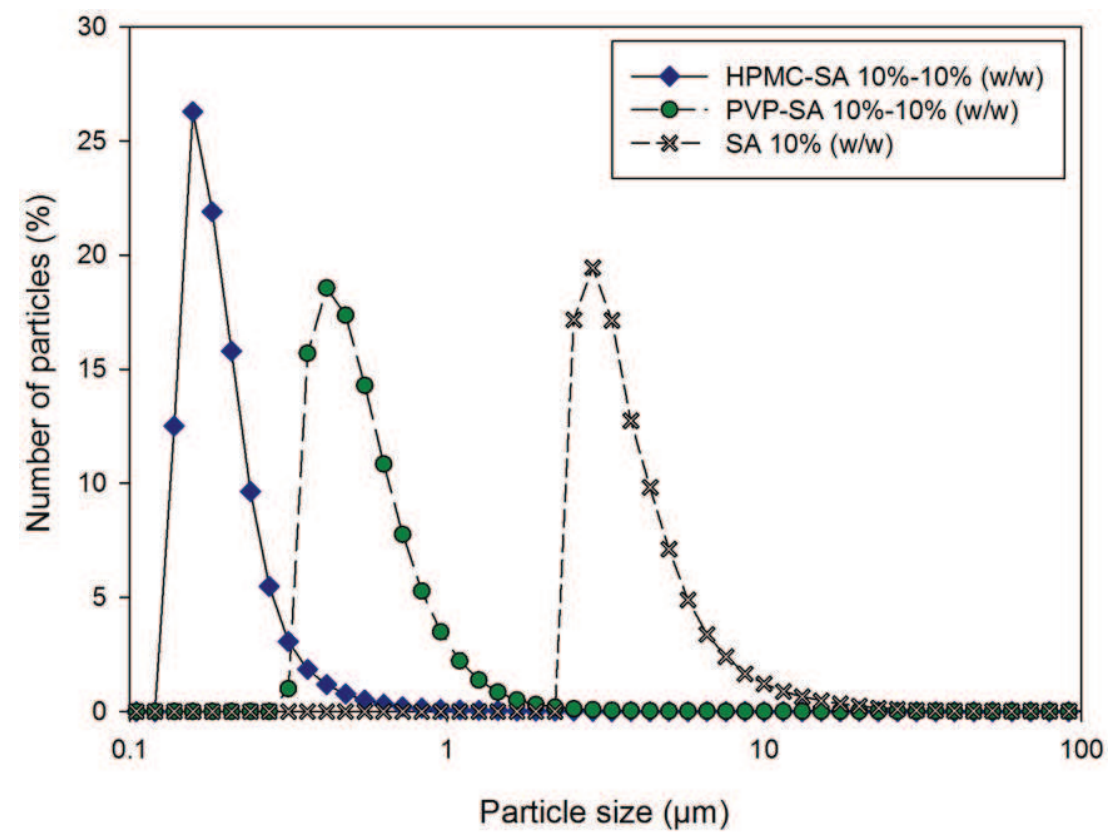

Fig. 16. Particle size distribution in number of HPMC-SA, PVP-SA and pure SA in water. HPMC: Hydroxypropyl-methylcellulose, PVP: Polyvinylpyrrolidone, SA: Stearic acid. 
Table 8

Granular properties in volume of the dispersions.

\begin{tabular}{lrrrrl}
\hline Sample & \multicolumn{1}{c}{$d_{10}(\mu \mathrm{m})$} & \multicolumn{1}{c}{$d_{50}(\mu \mathrm{m})$} & \multicolumn{1}{c}{$d_{90}(\mu \mathrm{m})$} & \multicolumn{1}{c}{$d_{32}(\mu \mathrm{m})$} & \multicolumn{1}{c}{$C v$} \\
\hline SA 10\% (w/w) & 81.32 & 387.27 & 684.62 & 105.93 & 1.56 \\
PVP-SA 10\%-10\% (w/w) & 2.42 & 41.78 & 135.42 & 6.72 & 3.18 \\
HPMC-SA 10\%-20\%(w/w) & 5.14 & 246.65 & 369.45 & 13.70 & 1.47 \\
HPMC-SA 10\%-10\% (w/w) & 0.22 & 1.37 & 5.69 & 0.62 & 3.99 \\
HPMC-SA 10\%-2\% (w/w) & 0.16 & 0.26 & 0.55 & 0.25 & 1.50 \\
\hline
\end{tabular}

SA: Stearic acid, PVP: Polyvinylpyrrolidone, HPMC: Hydroxypropyl-methylcellulose.

comes out higher than for a typical fluid, when expressed in physical units. As follows from Groot and Warren [13], it is possible to increase the dissipation strength to achieve exact agreement with the experiments. Unfortunately, this requires a much smaller time step to be able to integrate the higher friction forces. Furthermore, since we are mainly looking for qualitative results and considering that the structure of the materials in the DPD simulation is not affected by the dissipation strength, we chose to keep the default values of the dissipation strength proposed by Groot and Warren [13].

According to Eq. (25), a steeper slope in the diffusion curve indicates fast diffusion. HPMC reaches steady state faster than MCC and PVP and have lower first peak (Fig. 14), meaning that trapping SA molecules by HPMC polymer is easier than by the other polymers. This suggests that HPMC is a better stabilizing agent for SA than MCC and PVP.

\subsubsection{Affinity predictions obtained from DPD simulations}

The adherence of a material on the surface of a second material requires good affinity between them. Strong affinity between two materials in an aqueous system translates into thicker layer on the surface of the stronger cohesive material [4]. Jarray et al. extended the work of adhesion and the tensile strength equations originally formalized by Gardon [43] to ternary systems, and then predicted the affinity between different compounds in aqueous systems. Tables 7 shows the affinity between the materials predicted using the DPD simulation results, and compares them with Jarray et al.'s [4] work of adhesion $W_{\text {Adhesion }}$ predictive model and tensile strength $\sigma_{\text {tensile }}$ predictive model.

In Table 7, the letter "O" implies that compound A tend to adhere on compound $B$ in water, the letter " $X$ " implies the opposite (i.e. compound $B$ tend to adhere on compound $A$ in water), and the letter " $M$ " means compounds $\mathrm{A}$ and $\mathrm{B}$ are mixed in water.

From Table 7, we can see that the DPD simulations and Jarray et al.'s [4] work of adhesion approach tend to give similar predictions. All approaches predict that MCC and HPMC adhere on the surface of SA when they are dispersed in water. The difference between the approaches occurs for PVP-SA. The reason could be that the cohesion work of SA in water is underestimated when using Gardon's correlation in the case of the work of adhesion approach (see our previous work in Ref. [4] for more details).

\subsection{Experimental results}

\subsubsection{Particle size distribution}

The prepared samples were subjected to laser diffraction particle size analyzer with a Master Sizer (MALVERN). The particle size analyses, reported throughout this study, are the average of three successive laser diffraction runs. Particle size analysis of aqueous solutions of PVP-SA

Table 9

Granular properties in number of the dispersions.

\begin{tabular}{lllll}
\hline Sample & $d_{10}(\mu \mathrm{m})$ & $d_{50}(\mu \mathrm{m})$ & $d_{90}(\mu \mathrm{m})$ & $C v$ \\
\hline SA 10\% (w/w) & 2.33 & 3.19 & 4.9 & 0.81 \\
PVP-SA 10\%-10\% (w/w) & 0.33 & 0.47 & 0.82 & 1.04 \\
HPMC-SA 10\%-20\% (w/w) & 2.31 & 2.84 & 4.7 & 0.85 \\
HPMC-SA 10\%-10\% (w/w) & 0.13 & 0.16 & 0.27 & 0.87 \\
HPMC-SA 10\%-2\% (w/w) & 0.12 & 0.15 & 0.24 & 0.8 \\
\hline
\end{tabular}

SA: Stearic acid, PVP: Polyvinylpyrrolidone, HPMC: Hydroxypropyl-methylcellulose.

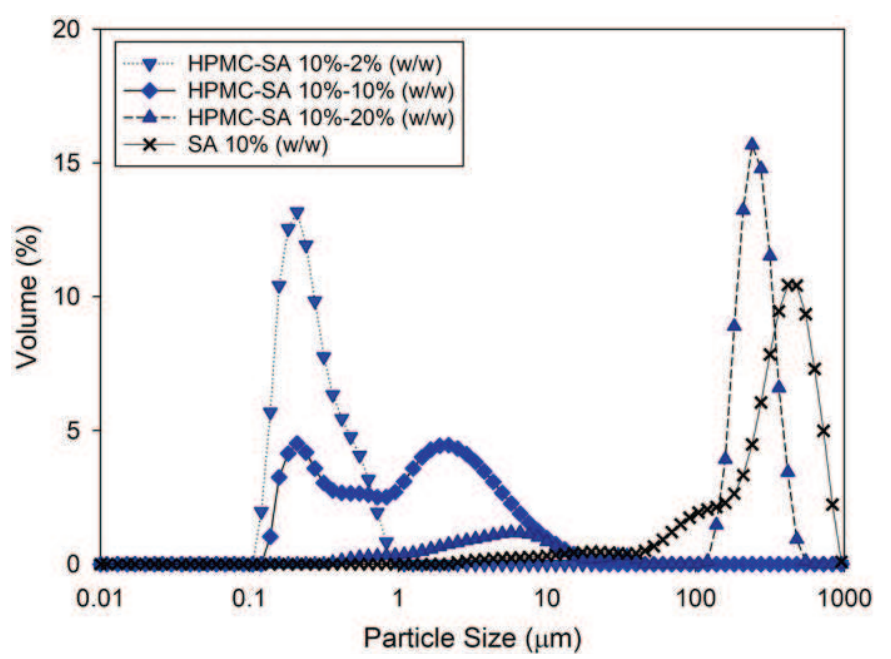

Fig. 17. Particle size distribution in volume of HPMC-SA under different percentages of SA. HPMC: Hydroxypropyl-methylcellulose, SA: Stearic acid.

and HPMC-SA show only the particle size distribution of SA in the mixtures. Particle size distribution in number is calculated using particle size distribution in volume results, on the assumption that the particles are spherical.

Particles size distribution in number and in volume of aqueous solution of pure SA (10\% (w/w)), HPMC-SA (10\%-10\% (w/w)) and PVP-SA $(10 \%-10 \%(w / w))$ are shown in Figs. 15 and 16 respectively. Tables 8 and 9 show the granular properties of each suspension in volume and in number respectively. $d_{10}$ and $d_{90}$ are the particle sizes below which $10 \%$ and $90 \%$ of the particles respectively belong, and $d_{32}$ is the surface weighted mean. Granular properties of the dispersions in number and in volume are also shown in Table 8. The coefficient $C v$ in Tables 8 and 9 measure the width of the distribution. The narrower the distribution, the lower the $C v$ value.

The SA (10\% (w/w)) curve in Fig. 15 shows that the majority of SA agglomerates have a size above $5 \mu \mathrm{m}$ with a mean diameter of $d_{50}=$ $387.269 \mu \mathrm{m}$. SA is insoluble in water and its hydrophobic character favors the agglomeration of SA molecules, thus, forming large cluster. Regarding the HPMC-SA (10\%-10\%) mixture, the distribution is multimodal and wider $(C v=3.99)$ with fine particles around $0.3 \mu \mathrm{m}$ and the mean diameter in volume is $d_{50}=1.369 \mu \mathrm{m}$. This means that the SA crystals are stabilized by the HPMC polymer with formation of some small agglomerate with a size between 1 and $20 \mu \mathrm{m}$. However, in the case of the PVP-SA mixture, the mean diameter is higher $\left(d_{50}=41.78 \mu \mathrm{m}\right)$ compared to the HPMC-SA case and the curve is in a higher particle diameter range (between $0.5 \mu \mathrm{m}$ and $900 \mu \mathrm{m}$ ). According to Fig. 16 showing the particle size distribution in number, the majority of SA particles for PVP are below $1 \mu \mathrm{m}$. This means that PVP is able to partially stabilize SA but it is not as effective as HPMC. According to our DPD simulations, this can be attributed to the low adsorption strength of PVP on the SA surface and to the thin layer formed by PVP around SA agglomerate.

The effect of SA percentage on the particle size distribution in number and in volume is shown in Figs. 17 and 18 respectively. The curves show that the control of SA agglomeration by HPMC is limited to SA percentages below $20 \%$. At $2 \%(\mathrm{w} / \mathrm{w})$ of $S A$, the curve is narrow and the agglomerates are monodisperse. HPMC fully stabilizes SA giving rise to the smallest particles. As the percentage of SA increases, the median particle size in volume increases significantly from $0.26 \mu \mathrm{m}$ to $246.65 \mu \mathrm{m}$ and the size distribution curve shifts to higher distribution sizes (Fig. 17). This traduces a formation of big SA agglomerates especially at 20\% (w/w) of SA where the solution shows narrower distribution curve at higher particles sizes $(C v=1.47)$. At this point, any other addition of SA particles will not have a noticeable effect on 


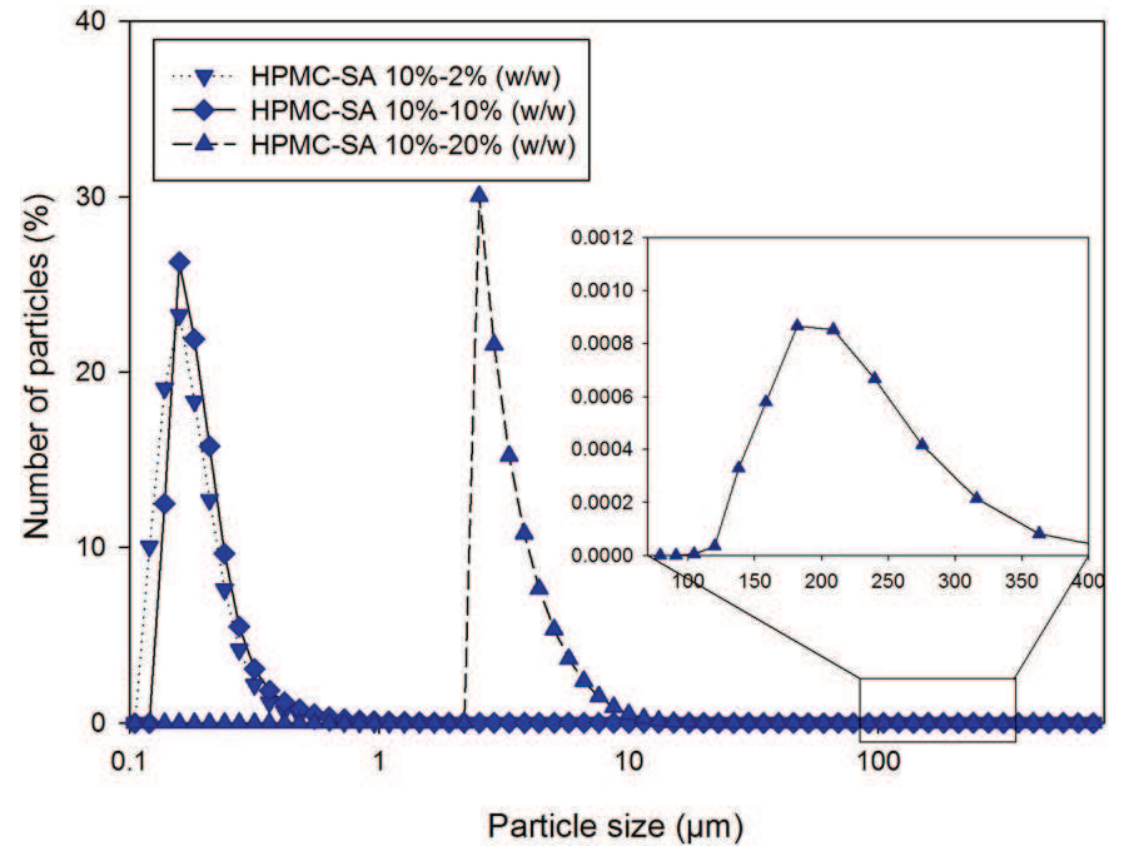

Fig. 18. Particle size distribution in number of HPMC-SA under different percentages of SA. HPMC: Hydroxypropyl-methylcellulose, SA: Stearic acid.

the particle size distribution. Overall, even though DPD simulations are in the germ scale, experimental and DPD results share the same tendencies.

\subsubsection{Cryogenic-SEM results}

To distinguish between HPMC and SA structure, two cryofixated samples were observed using transmission electron microscopy (SEM), the first sample contains 10\% of HPMC in water and the second one contains $10 \%$ of SA in water.

From a glance at the SEM images presented in Fig. 19, we can distinguish between SA and HPMC. SA has the form of crystalline needles that form large agglomerate in water and their size is around $50 \mu \mathrm{m}$ (Fig. 19(c)), while HPMC becomes amorphous and forms transparent solution (Fig. 19(a)) which makes it difficult to distinguish between HPMC and water.
When the samples are sublimated (Fig. 19(b) and (d)), we notice that HPMC-water architecture shows a perforated structure designed by the sublimated ice crystals templates. Cryofixation using pasty nitrogen is a relatively slow freezing process that generates ice crystals inside the samples, consequently, inner parts of HPMC-water mixture freeze slower than the outer parts, and therefore, exhibit larger pores after sublimation. To avoid the formation of crystals we also used cryo-fixation using liquid ethane. Samples frozen using liquid ethane are shown in Fig. 20(b), (d) and (f).

SEM images confirm the previous statements obtained by particle size distribution. In Fig. 20, HPMC-SA mixed in aqueous system was subjected to cryo-fixation first using pasty then using liquid ethane. Then they were sublimated. The structure of SA crystals has a significant change in the presence of HPMC. When HPMC-SA sample is frozen using pasty nitrogen (Fig. 20(a), (c) and (e)), HPMC shows pores in

HPMC $10 \%(w / w)$ in water, pasty nitrogen

a) Before sublimation
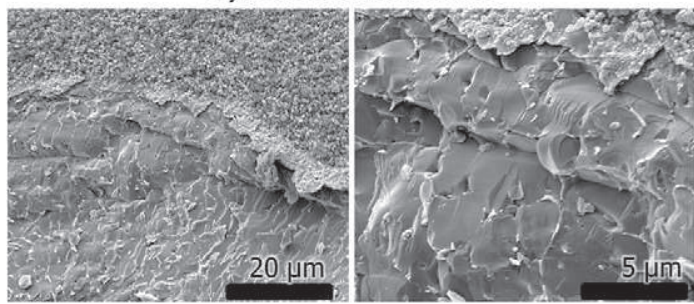

b) After sublimation

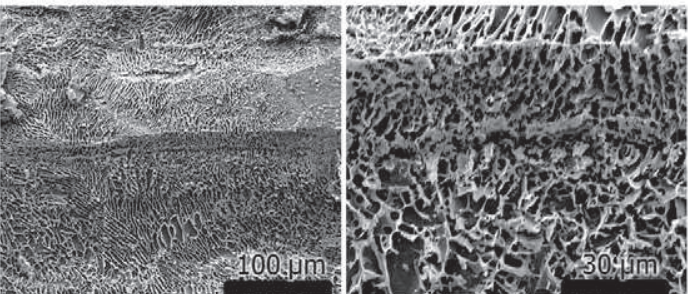

SA $10 \%(w / w)$ in water, pasty nitrogen

c) Before sublimation

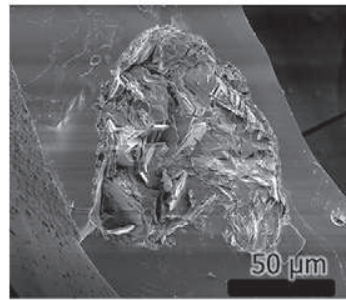

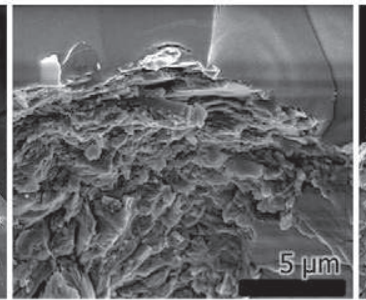

d) After sublimation
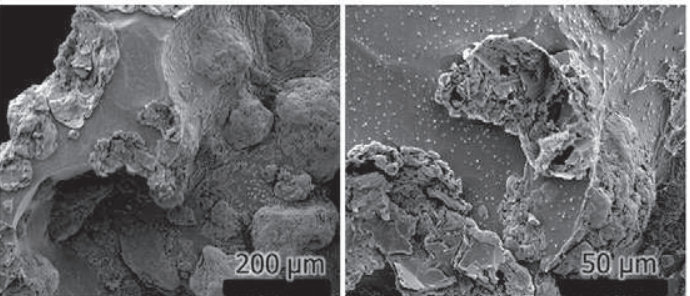

Fig. 19. SEM micrographs of HPMC (top) and SA (bottom) in water before and after sublimation, cryofixated using pasty nitrogen. HPMC: Hydroxypropyl-methylcellulose, SA: Stearic acid. 
HPMC-SA $10 \%-20 \%(w / w)$ in water, inner structure

a) Pasty nitrogen

b) Liquid ethane

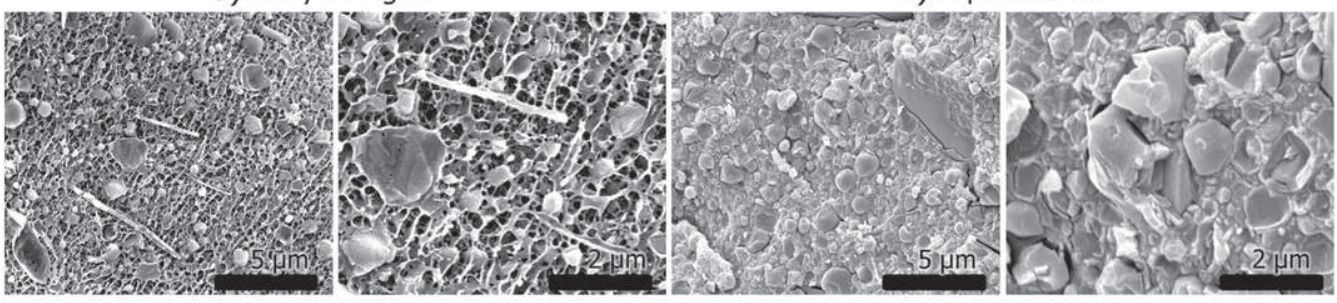

HPMC-SA $10 \%-10 \%(w / w)$ in water, inner structure

c) Pasty nitrogen

d) Liquid ethane
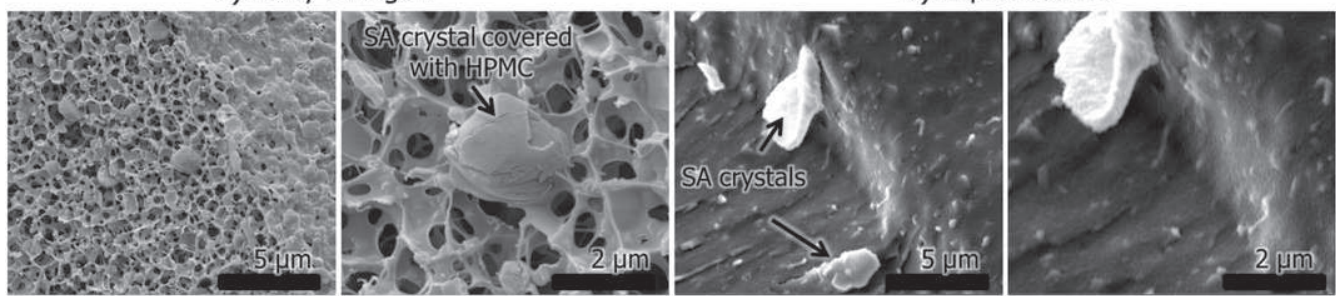

HPMC-SA $10 \%-2 \%(w / w)$ in water, inner structure

e) Pasty nitrogen

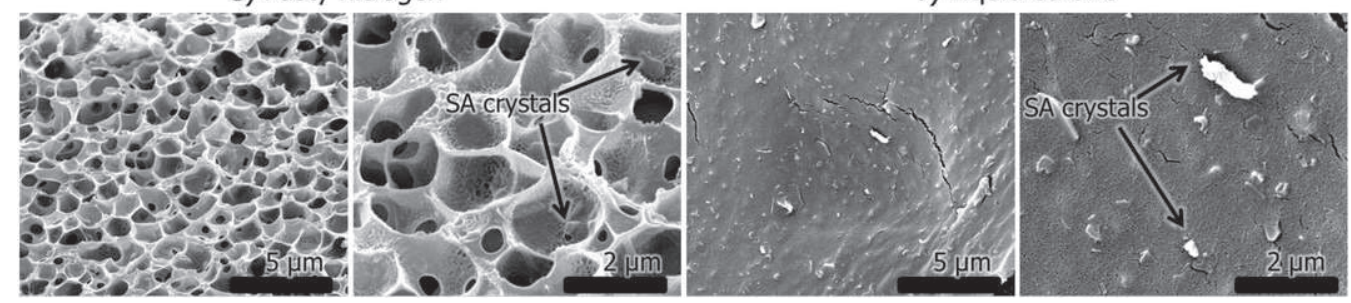

Fig. 20. SEM micrographs of HPMC-SA in water under different percentages of SA and taken after sublimation. HPMC: Hydroxypropyl-methylcellulose, SA: Stearic acid.

the micrometer scale, patterned by the ice crystals. When using liquid ethane as a freezing medium (Fig. 20(b), (d) and (f)), the pore size becomes significantly small and cannot be seen in the edge of the fractured sample. In Fig. 20(a), (c) and (e), we can see that the pores size depends also on the SA contents. An increase in the SA content in the mixture increases the pore size. When using pasty nitrogen, SA crystals can be seen inside the HPMC pores network and they are covered by the HPMC.
Alternatively, when using liquid ethane, SA white crystals are more distinguishable, some of them are covered by HPMC, and their distribution in the HPMC-water blend is more noticeable.

As shown in Fig. 20, the size and distribution of SA particles within the suspension varies under different amounts of SA. When the SA weight percentage is up to $20 \%$, SA agglomerates become notably large and seems more polydisperse; which destabilizes the dispersion

PVP-SA $10-10 \%(w / w)$, liquid ethane, inner structure

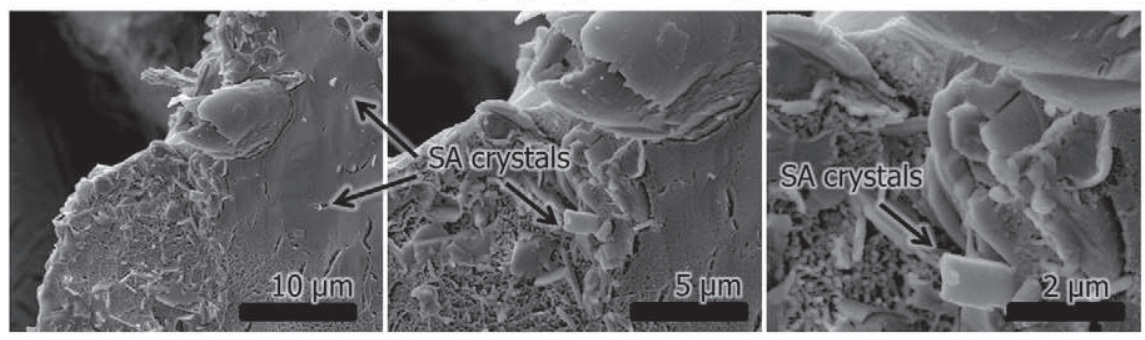

PVP-SA $10-10 \%(w / w)$, liquid ethane, surface

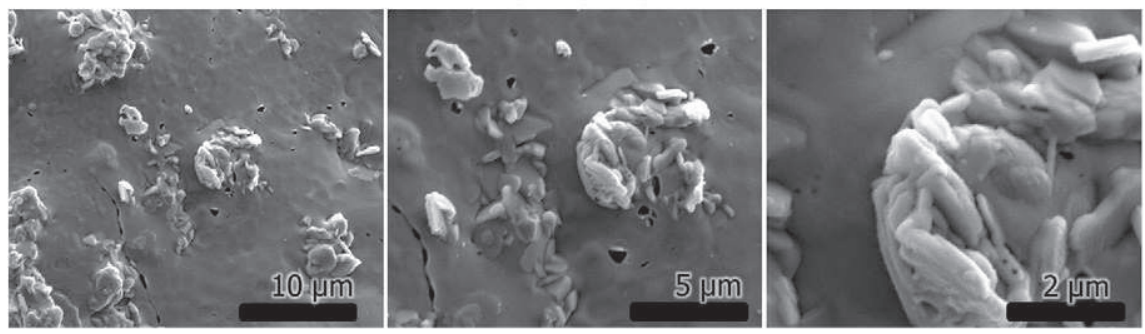

Fig. 21. SEM micrographs of PVP-SA in water taken after sublimation. PVP: Polyvinylpyrrolidone, SA: Stearic acid. 
(Fig. 20(a) and (b)). The likely reason for the re-arrangement of the structure of SA agglomerate is that the amount of HPMC is insufficient to reduce the free energy associated with the SA crystallites. Therefore HPMC becomes unable to prevent SA agglomeration, causing the small SA aggregates to adhere on the surface of large SA agglomerate and thus, their growth. We inferred from simulation results that a HPMCSA ratio 1:2 was not enough to allow an efficient stabilization of the aqueous SA suspension. On the other hand, when the SA weight percentage is $2 \%$, SA crystals are evenly dispersed in the HPMC suspension and their size is below $1 \mu \mathrm{m}$ in diameter (Fig. 20(e) and (f)). HPMC is very well anchored on the surface of the SA agglomerate and covers it with a hatching textured film that resembles dried soil (Fig. 20(c) and (e)). SA crystals are therefore trapped in the HPMC network. This allows the stabilization of SA agglomerates whose size is near $1 \mu \mathrm{m}$ in diameter. A similar conclusion was reached in the DPD simulation section where we showed that at $2 \%(\mathrm{w} / \mathrm{w})$ of SA, $50 \%$ of HPMC forms a thick layer around SA beads and 50\% of HPMC beads form a network inside SA core. This also corresponds to the Malvern particle distribution analysis shown in Fig. 17 where samples with lower SA contents have the lowest mean particle diameter.

Fig. 21 presents the SEM images of PVP-SA (10\%-10\%) sample. In the inner part of the sample (Fig. 21(a)), big SA agglomerates as well as some small SA crystals (in white) can be seen. This means that some of the small primary SA particles are stabilized by PVP. This can be confirmed by the particle size distribution shown in Figs. 15 and 16 where there is SA particles below $1 \mu \mathrm{m}$ in size. We also noticed that in the surface of the PVP-SA sample, SA crystals form bigger agglomerates with irregular shape (Fig. 21(b)). Non-stabilized SA agglomerates migrate to the surface of the sample and form larger agglomerates. We may deduce that PVP is not as effective stabilizer as HPMC but it is able to partially stabilize SA. Similarly, in the simulation section we observed that PVP forms a thin layer around SA beads and it diffuses mainly on the outer core of SA agglomerate without forming a network.

\section{Conclusion}

In this study, we proposed a mesoscale "coarse-grain" model for hydroxypropyl-methylcellulose (HPMC), polyvinylpyrrolidone (PVP), microcrystalline cellulose (MCC), polyethylene glycol (PEG) and stearic acid (SA). DPD method was applied to the coarse grain model and dynamic simulations were launched, allowing one to describe the structure of colloidal suspensions composed of the aforementioned polymers. Interfacial energy of pure compounds obtained from DPD simulations are close to the experimental values. We have examined polymer-SA interactions with particular emphasis on the percentage of polymer that diffuses inside SA agglomerate. DPD simulation results were further analyzed using the structure factor and the diffusion coefficient. The results show that our "coarse grain" model is able to reproduce some structural features of aqueous colloidal formulations in the germ scale.

According to DPD results, at low percentages of SA (below 10\% $(w / w))$, HPMC completely covers SA and forms a network that diffuses deeply through SA. At higher SA load a tubular structure is obtained and there is not enough HPMC to cover the SA core and to penetrate inside it. MCC interposes in the outer surface of SA agglomerate without diffusing inside them. PVP shows an opposite behavior comparing to MCC and high amount of PVP beads diffuses through SA particles and a tubular structure is obtained. The affinity results between the materials obtained through DPD simulation are similar to those obtained by the predictive models of Jarray et al. [4] based on the work of adhesion and the tensile strength.

Experimental results show similar trends. At low SA percentage (below $10 \%(\mathrm{w} / \mathrm{w})$ ), HPMC fully stabilizes SA which gave rise to the smallest SA particles. Increasing SA percentage led to bigger SA agglomerates and unstable polymeric suspension. When using PVP as a stabilizer, the median size increases but some of the SA particles are below $1 \mu \mathrm{m}$ in diameter, meaning that PVP is able to partially stabilize SA. SEM images reveal that HPMC surrounds SA agglomerates with a hatching textured film and anchors on their surface, thus preventing their agglomeration. Upon increasing the SA percentage, larger SA agglomerates are seen in the SEM images.

\section{List of symbols}

$\begin{array}{ll}a_{i j} & \text { Interaction parameter between bead } i \text { and bead } j \\ C_{r} & \text { The harmonic spring constant } \\ C_{n} & \text { Characteristic ratio of the polymer } \\ C v & \text { Width of the distribution } \\ D & \text { Diffusivity coefficient } \\ f_{i} & \text { Sum of the forces acting on the bead } i \\ F_{i j} & \text { Force exerted by a bead } i \text { on a second bead } j \\ F^{C} & \text { Conservative repulsive force } \\ F^{D} & \text { Dissipative force } \\ F^{R} & \text { Random force } \\ F^{S} & \text { Bonding spring force } \\ H & \text { Heaviside function } \\ k_{B} & \text { Boltzmann constant } \\ M_{w} & \text { Molecular weight of the polymer } \\ M_{m} & \text { Molecular weight of the monomer } \\ m & \text { Mass } \\ n_{D P D} & \text { DPD number } \\ N_{m} & \text { Coarse-grain number } \\ N_{B e a d s} & \text { Number of beads } \\ P & \text { Pressure } \\ r_{c} & \text { Cutoff radius } \\ r_{i} & \text { Position of the bead } i \\ R_{\text {equivalent }} & \text { Equivalent agglomerate radius } \\ S & \text { Structure factor } \\ T & \text { Temperature } \\ V_{i} & \text { Volume of the bead } i \\ v_{i} & \text { Velocity of the bead } i \\ W_{\text {Adhesion }} & \text { Work of adhesion } \\ \rho & \text { Number density } \\ e & \text { Density } \\ \sigma_{\text {tensile }} & \text { Tensile strength } \\ \delta & \text { Solubility parameter } \\ \xi_{i j} & \text { Random parameter } \\ \partial & \text { Parameter of dissipation } \\ \alpha & \text { Adjustment parameter } \\ \chi_{i j} & \text { Flory-Huggins parameter } \\ \omega & \text { Weight function } \\ \kappa^{-1} & \text { Compressibility factor } \\ \gamma & \text { Interfacial energy } \\ \Delta & \text { Dirac function } \\ - & \text { Upper-script that denotes the property in DPD units } \\ & \end{array}$

\section{Appendix A}

By combining the compressibility of the DPD model with that of the real system, Groot and Warren [13] were able to relate the compressibility factor $\kappa^{-1}$ to the parameter of repulsion $a_{i i}$ between the same type of beads:

$$
\begin{aligned}
\left(\kappa^{-1}\right) & =\left(\bar{\rho}_{\text {molecule }} k_{B} T \kappa_{T}\right)^{-1}=\frac{1}{k_{B} T}\left(\frac{\partial P}{\partial \rho_{\text {molecule }}}\right)_{T} \\
& =\frac{r_{c}^{3}}{k_{B} T}\left(\frac{\partial P}{\partial \bar{\rho}_{\text {molecule }}}\right)_{T}
\end{aligned}
$$




$$
\left(\bar{\kappa}^{-1}\right)=1+\frac{2 \alpha a_{i i} \rho r_{c}^{4}}{k_{B} T}=\left(\frac{\partial \bar{P}}{\partial \bar{\rho}}\right)_{T}=\frac{r_{c}^{3}}{k_{B} T}\left(\frac{\partial P}{\partial \bar{\rho}}\right)_{T}
$$

where $P$ is the pressure, $T$ the temperature, $\alpha$ is an adjustment parameter equal to $0.101( \pm 0.001)$, and $\kappa_{T}$ the isothermal compressibility of the compound. At the same time, we must keep in mind that this compressibility factor varies linearly with the coarse-grain number $N_{m}$, a detail originally passed over by Groot and Warren [13], and recently rectified by Groot and Rabone [14]. In the DPD simulations, the compressibility must be equal to that of the real system:

$$
\left(\bar{\kappa}^{-1}\right)=\frac{r_{c}^{3}}{k_{B} T}\left(\frac{\partial P}{\partial \bar{\rho}}\right)_{T}=\left(\kappa^{-1}\right)=\frac{r_{c}^{3}}{k_{B} T}\left(\frac{\partial P}{\partial \bar{\rho}_{\text {molecule }}}\right)_{T} .
$$

Substitution from Eq. (8), we obtain:

$$
\frac{1}{k_{B} T}\left(\frac{\partial P}{\partial \bar{\rho}}\right)_{T}=\frac{1}{k_{B} T}\left(\frac{\partial \bar{\rho}_{\text {molecule }}}{\partial \bar{\rho}}\right)_{T}\left(\frac{\partial P}{\partial \bar{\rho}_{\text {molecule }}}\right)_{T}=\frac{N_{m}}{k_{B} T}\left(\frac{\partial P}{\partial \bar{\rho}_{\text {molecule }}}\right)_{T} .
$$

Combination with Eq. (A.2) gives:

$$
\left(\kappa^{-1}\right)=\frac{\left(\bar{\kappa}^{-1}\right)}{N_{m}}=\frac{1}{N_{m}}+\frac{2 \alpha a_{i i} \rho r_{c}^{4}}{k_{B} T N_{m}}
$$

Under standard conditions, the dimensionless compressibility of water is $\left(\kappa^{-1}\right)=15.9835 \approx 16$, hence, it becomes possible to determine the repulsion parameter $a_{i i}$ :

$a_{i i}=\left(16 N_{m}-1\right) \frac{k_{B} T}{2 \alpha \rho r_{c}^{4}}$

\section{References}

[1] R.C. Rowe, Adhesion of film coating to tablet surfaces - a theoretical approach based on solubility parameters, Int. J. Pharm. (1988) 219-222.

[2] J. Barra, F. Lescure, F. Falson-Rieg, E. Doelker, Can the organization of a binary mix be predicted from the surface energy, cohesion parameter and particle size of its components? Pharm. Res. 15 (1998) 1727-1736.

[3] M. Benali, Prédiction des interactions substrat-liant lors de la granulation : Etude expérimentale dans un mélangeur à fort taux de cisaillement, approches thermodynamiques par simulation moléculaire, 2006.

[4] A. Jarray, V. Gerbaud, M. Hemati, Prediction of solid-binder affinity in dry and aqueous systems: Work of adhesion approach vs. ideal tensile strength approach, Powder Technol. 271 (2015) 61-75.

[5] D.H. Napper, Polymeric Stabilization of Colloidal Dispersions, Academic Press, London, 1983.

[6] G.O. Phillips, P.A. Williams, Handbook of Hydrocolloids, second ed., 2009

[7] H. Koelmans, J.T.C. Overbeek, Discussions of the Faraday Society, 195418.

[8] D.-J. Walbridge, J.A. Waters, Rheology of sterically stabilized dispersions of polymethyl methacrylate) in aliphatic hydrocarbons, Discuss. Faraday Soc. 42 (1966) 294-300

[9] P.J. Hoogerbrugge, J.M.V.A. Koelman, Simulating microscopic hydrodynamic phenomena with dissipative particle dynamics, Europhys. Lett 30 (4) (1992) 191-196.

[10] P. Langevin, On the theory of Brownian motion, C. R. Acad. Sci. (Paris) 146 (1908) 530-533.
[11] P. Español, Dissipative particle dynamics revisited, SIMU Newsletter 4 (2002) 59-77.

[12] K.B. Lipkowitz, D.B. Boyd, Reviews in Computational Chemistry, 27Wiley, 2010.

[13] R.D. Groot, P.B. Warren, Dissipative particle dynamics: bridging the gap between atomistic and mesoscopic simulation, J. Chem. Phys. 107 (1997) 4423-4435.

[14] R.D. Groot, K.L. Rabone, Mesoscopic simulation of cell membrane damage, morphology change and rupture by nonionic surfactants, Biophys. J. 81 (2001) 725-736.

[15] E.G. Flekkoy, G. Wagner, J. Feder, Hybrid model for combined particle and continuum dynamics, Europhys. Lett. 52 (3) (2000) 271-276.

[16] P. Español, M. Serrano, I. Zuniga, Coarse-graining of a fluid and its relation to dissipative particle dynamics and smoothed particle dynamics, Int. J. Mod. Phys. C 8 (1997) 899-908.

[17] S. Trofimov, Thermodynamic consistency in dissipative particle dynamicsPh.D. thesis TechnischeUniversiteit Eindhoven, 2003.

[18] J.-P. Hansen, L. Verlet, Phase transitions of the Lennard-Jones system, Phys. Rev. 84 (1969) 151-161.

[19] J.-P. Hansen, D. Schiff, Influence of interatomic repulsion on the structure of liquids at melting, Mol. Phys. 25 (6) (1973) 1281-1290.

[20] I.V. Pivkin, G.E. Karniadakis, Controlling density fluctuations in wall bounded dissipative particle dynamics systems, Phys. Rev. Lett. 96 (2006) 206001.

[21] J.H. Hildebrand, The solubility of nonelectrolytes, third ed. Reinhold Pub. Corp, New York, 1950.

[22] P.J. Flory, Thermodynamics of high polymer solutions, J. Chem. Phys. 10 (1942) 51-61.

[23] A. Maiti, S. McGrother, Bead-bead interaction parameters in dissipative particle dynamics: Relation to bead-size, solubility parameter, and surface tension, J. Chem. Phys. 120 (2004) 1594-1601.

[24] K.P. Travis, M. Bankhead, K. Good, S.L. Owens, New parametrization method for dissipative particle dynamics, J. Chem. Phys, 127 (2007) 014109.

[25] Biovia, Material Studio Suite release 7, http://accelrys.com/products/materials-studio/index.html2013.

[26] H. Irisa, T. Yokomine, Determination of DPD model aiming biomedical application, Int. J. Soft Comput. Biomed. Hum. Sci. 15 (2) (2010) 27-32.

[27] H. Guo, X. Qiu, J. Zhou, Self-assembled core-shell and Janus microphase separated structures of polymer blends in aqueous solution, J. Chem. Phys 139 (2013) 084907.

[28] H. Yamamoto, HSPiP V.3.1 Software, http://www.hansen-solubility.com/HSPiP. html2010.

[29] H. Sun, COMPASS: An ab initio force-field optimized for condensed-phase applications overview with details on alkane and benzene compounds, J. Phys. Chem. B 102 (1998) 7338-7364.

[30] J. Bicerano, Prediction of Polymer Properties, third ed. CRC Press, New York, 2002.

[31] J.H. Irving, J.G. Kirkwood, The statistical mechanical theory of transport processes. IV. The equations of hydrodynamics, J.Chem.Phys. 18 (6) (1950) 817-829.

[32] R.C. Rowe, Interactions in coloured powders and tablet formulations: a theoretical approach based on solubility parameters, Int. J. Pharm. 53 (1989) 47-51.

[33] M. Brogly, A. Fahs, A. Bistac, Surface properties of new-cellulose based polymer, coatings for oral drug delivery systems, Polym. Prepr 52 (2) (2011) 1055-1056.

[34] R.C. Rowe, Polar/non-polar interactions in the granulation of organic substrates with polymer binding agents, Int. J. Pharm. 56 (1989) 117-124.

[35] G. Wypych, Handbook of Antiblocking, Release, and Slip Additives, third ed. ChemTec Publishing, 2014.

[36] L.P. Demajo, D.S. Rimai, L.H. Sharpe, Fundamentals of Adhesion and Interfaces, CRC Press, 2000.

[37] B. Vincent, The effect of adsorbed polymers on dispersion stability, Adv. Colloid Interf. Sci. 4 (1974) 193-277.

[38] F. Laboulfie, Depôt en couche mince d'un multi-materiau à la surface de particules solides : application à l'enrobage de particules alimentaires, INPT, Toulouse, 2013.

[39] P. Müller, A. Russell, J. Tomas, Influence of binder and moisture content on the strength of zeolite 4A granules, Chem. Eng. Sci. 126 (2015) 204-215.

[40] K.L. Mittal, P. Kumar, Emulsions, Foams, and Thin Films, Taylor \& Francis Inc, 2000

[41] M.P. Allen, D.J. Tildesley, Computer Simulation of Liquids, Oxford Science Publications, Clarendon Press, 1987.

[42] A. Einstein, Über Die von der Molekularkinetischen Theorie der Wärme Geforderte Bewegung von in Ruhen-den Flüssigkeiten Suspendi, Physik 322 (8) (1905) 549-560.

[43] L. Gardon, Variables and interpretation of some destructives cohesion and adhesion tests, Treatise on Adhesion and Adhesives 1967, pp. 269-324. 